\title{
Photometric and morphological analysis of UCM galaxies. I. Observations and reductions. Morphological classifications ${ }^{\star, \star \star}$
}

\author{
A.G. Vitores ${ }^{1,2}$, J. Zamorano ${ }^{1}$, M. Rego ${ }^{1}$, O. Alonso ${ }^{1}$ and J. Gallego ${ }^{1}$ \\ ${ }^{1}$ Dpto Astrofísica, Facultad CC Físicas, Universidad Complutense, E-28040 Madrid, Spain \\ 2 EUIT Industrial, Universidad Politécnica, E-28012 Madrid, Spain
}

Received May 31; accepted December 18, 1995

\begin{abstract}
We present the results of Thuan \& Gunn $r$ CCD imaging observations of the Universidad Complutense de Madrid (UCM) sample of emission-line galaxies (ELGs), selected by the presence of $\mathrm{H} \alpha$ emission in low-resolution objective prism spectra. In this work we characterize photometrically and morphologically a total of 212 objects from the UCM survey. This Paper presents the observations and basic reductions, and lists a set of photometric parameters calculated for each UCM object. In addition, for the first time in a ELGs sample, we have determined the morphological classification of the objects using simultaneously five different criteria.
\end{abstract}

Key words: surveys — galaxies: fundamental parameters - galaxies: general — galaxies: photometry — atlases

\section{Introduction}

The UCM $\mathrm{H} \alpha$ objective-prism survey is being carried out with the main purposes of identifying and studying new young, low metallicity galaxies and quantifying the properties of the star formation in the local universe. To achieve these goals it is necessary to characterize photometrically and spectroscopically the emission-line galaxies (ELGs) of the UCM sample. The specific details of the UCM survey have already been summarized in the first and second lists (Zamorano et al. 1994; 1995) of our exploration. Since the spectroscopic results of the sample are presented in Gallego et al. (1995), we will concentrate here in the photometric characteristics of the survey.

Although some important samples of ELGs selected from objective prism plates have been imaged in the blue, e.g. the University of Michigan (UM) survey (Salzer et al. 1989) or in the red spectral band, e.g. the Wasilewski (Was) survey (Bothun et al. 1989), a systematical morphological classification of the selected objects has never been undertaken. Here, for the first time, we characterize morphologically a sample of objects selected by the presence of emission lines in their objective prism spectra.

Send offprint requests to: M. Rego

${ }^{\star}$ Based on observations collected at the German-Spanish Astronomical Center, Calar Alto, Spain, operated by the MaxPlanck-Institut für Astronomie (MPIA), Heidelberg, jointly with the Spanish National Commision for Astronomy

${ }^{\star \star}$ Tables 2-7 are only available at the CDS via ftp 130.79.128.5
In this Paper, we analyse a sample of 212 ELGs candidates extracted from UCM lists 1 and 2 (Zamorano et al. 1994, 1995). The obtained CCD images have been used (1) to compute basic photometric properties (size and luminosity) of the UCM objects; (2) to obtain, via surface photometry, a set of specific photometric parameters (bulge+disk decomposition, concentration indices and mean surface brightnesses), and (3) to determine the Hubble type of these ELGs. In Paper II (Vitores et al. 1995) a statistical analysis of the UCM sample will be presented: In particular, we will make a comparison with other samples of normal and emission-line galaxies and we will analyse the possible influence of the environment in the presence and nature of ELGs.

\section{Observations and reductions}

\subsection{Observations}

A set of CCD images (including bias,dark, flat-field, calibration stars, UCM objects and galaxies for comparison) was acquired through a Thuan \& Gunn (1976) $r$ filter. The $2.2 \mathrm{~m}$ and the $3.5 \mathrm{~m}$ telescopes of the Calar Alto GermanSpanish Observatory (Almería, Spain) were used to perform the observations during eight runs from December 1988 to January 1992. The $r$ filter was chosen since it includes the $\mathrm{H} \alpha+[\mathrm{NII}]$ blend, the basic feature used for the selection in the IIIaF+RG630 objective prism plates. Most of the images were taken at the Cassegrain focus of the $2.2 \mathrm{~m}$ telescope to obtain a spatial scale suitable to the rather small sizes of the galaxies. Nevertheless, the 
faintest objects were observed at the prime focus of the $3.5 \mathrm{~m}$ telescope. In the case of the images taken with the RCA detector, an on-chip binning was made in order to increase the $S / N$ ratios. Table 1 summarizes the instrumental setups used during different observing runs. The typical seeing was $\sim 11^{\prime \prime} 5-2{ }^{\prime \prime} 0$. Some images, taken with worse seeing but in good photometric conditions, were only used for calibration purposes, being repeated during subsequent nights with better seeing conditions. All the images taken in the five non-photometric nights were also repeated. Exposure times ranged between $900 \mathrm{~s}$ and $1800 \mathrm{~s}$.

\subsection{Basic reduction and calibration}

Basic reduction of the data was made using the MIDAS (Munich Image Data Analysis System) software package, developed at ESO (European Southern Observatory). The CCD frames were debiased and flat-fielded by using dome and sky flats, and any dark current effect was removed in the subtraction of the sky level. The images taken during the run of June 1989 needed an additional processing in order to remove a remanent additive effect in some columns of the chip. The images were also cleaned for cosmic ray impacts. Due to the relative small surface of the chip covered by our galaxies, there is a large fraction of the detector free of objects which guarantees the determination of the sky level. After processing, the sky background of the images was flat to $1 \%-2 \%$ levels.

Finally, the magnitudes of the objects were measured using an interactive aperture photometry routine which allows to compute the flux of the objects using circular apertures with variable diameters, and subtracting the sky background level measured inside an aperture with the same size in clean regions, free of contamination. Then, the data were converted to photometric magnitudes using the extinction and zero point constants derived from observations of a set of standard stars from the lists of Thuan \& Gunn (1976) and Kent (1985a) taken along each night. Table 2 presents the apparent magnitudes (the listed errors include photometric calibrations and poissonian contributions) which have been converted to absolute magnitudes using $H_{0}=50 \mathrm{~km} \mathrm{~s}^{-1} \mathrm{Mpc}^{-1}$ and the redshift measured in the spectra, when available, by Gallego et al. (1995). These values were corrected for galactic extinction using $A_{r}=2.51 E(B-V)$, where $E(B-V)$ was determined from the Burstein \& Heiles (1982) maps at the galactic coordinates of each UCM object. The uncertainties in the zero points of the photometric calibrations are listed in the last column of Table 1.

In order to investigate the reliability of the estimated errors in the total magnitudes, we have analysed the consistency of repeated measurements. From the rms deviations of the values for 12 galaxies in common among different runs, we have estimated an external error of $0.065 \mathrm{mag}$. This agrees quite well with the expected error for a typ- ical galaxy of this subsample (0.068 mag). Furthermore, no systematic offset in the zero points between different runs has been observed. Therefore, and although the sample with repeated observations is scarce, we are confident that no systematic errors are involved.

As a further consistency test, we have compared our estimated total magnitudes with those derived by Bothun et al. (1989) for 10 galaxies from the Wasilewski sample (these objects were included in our runs for comparison purposes). Although we have not found any significative mean offset $(0.01 \mathrm{mag})$ between both samples, the rms dispersion around the mean $(0.48 \mathrm{mag})$ is significantly larger than our internal errors. Since Bothun et al. (1989) do not provide their error estimates, this large dispersion does not necessarily mean any additional error source in our data.

\subsection{Surface photometry reduction}

In order to characterize the specific morphology of the UCM objects it is necessary to analyse the spatial distribution of the light along the radius. To achieve this, we have reduced the bidimensional information of the CCD frames to a one-dimensional one by the determination of surface brightness profiles. The basic surface photometry reduction was performed with the program PROF (PROFiles) of the GASP (Galaxy Surface Photometry) package written by Cawson (1983) and modified by González-Serrano \& Pérez-Fournon (1991) and de Juan y Ruiz (1994). The intensity images were fitted by ellipses which center, ellipticity and position angle were allowed to vary. Intensity levels of each isophote were then converted to surface brightness, and radial profiles (using the equivalent radius, that is, the geometric mean of the semimajor and semiminor axes of each elliptical isophote) of surface brightness, position angle and ellipticity were derived.

\subsection{Fitting of the surface brightness profiles}

The equivalent surface brightness profiles were fitted by a sum of bulge+disk empirical laws. We have used the classical $r^{1 / 4}$ law for the bulge component (de Vaucouleurs 1948)

$$
\mu=\mu_{\mathrm{e}}+8.33\left(\left(\frac{r}{r_{\mathrm{e}}}\right)^{1 / 4}-1\right)
$$

and the exponential law for the disk component (Freeman 1970)

$$
\mu=\mu_{0}+1.0857\left(\frac{r}{d_{\mathrm{L}}}\right)
$$

Our decomposition technique is similar to the Schombert \& Bothun (1987) method (based on a $\chi^{2}$ minimization of the differences between the observed and the sum of the theoretical profiles), although we have introduced some modifications. Our procedure is the following: 
Table 1. Log-book of observations

\begin{tabular}{|c|c|c|c|c|c|c|c|c|}
\hline $\begin{array}{c}\text { date of } \\
\text { observation }\end{array}$ & telescope & focus & detector & $\begin{array}{l}\text { pixel size } \\
\quad(\mu \mathrm{m})\end{array}$ & $\begin{array}{l}\text { detector size } \\
\quad\left(\text { pixel }^{2}\right)\end{array}$ & $\begin{array}{c}\text { scale } \\
(" / \text { pixel })\end{array}$ & field & $\begin{array}{c}\text { rms } \\
\text { zero point }\end{array}$ \\
\hline 12 Dec. 1988 & $2.2 \mathrm{~m}$ & Cassegrain & RCA\#11 & 30.0 & $512 \times 320$ & 0.35 & $3.0 \times 1.9$ & 0.03 \\
\hline 13 Dec. 1988 & $2.2 \mathrm{~m}$ & Cassegrain & RCA\#11 & 30.0 & $512 \times 320$ & 0.35 & $3.0 \times 1.9$ & 0.03 \\
\hline 14 Dec. 1988 & $2.2 \mathrm{~m}$ & Cassegrain & $\mathrm{RCA \# 11}$ & 30.0 & $512 \times 320$ & 0.35 & $3.0 \times 1.9$ & 0.03 \\
\hline 29 Jun. 1989 & $2.2 \mathrm{~m}$ & Cassegrain & RCA\#11 & 30.0 & $512 \times 320$ & 0.35 & $3 ! 0 \times 1{ }^{\prime} \cdot 9$ & 0.16 \\
\hline 30 Jun. 1989 & $2.2 \mathrm{~m}$ & Cassegrain & $\mathrm{RCA \# 11}$ & 30.0 & $512 \times 320$ & 0.35 & $3.0 \times 1.9$ & 0.04 \\
\hline 1 Jul. 1989 & $2.2 \mathrm{~m}$ & Cassegrain & RCA\#11 & 30.0 & $512 \times 320$ & 0.35 & $3.0 \times 1.9$ & 0.03 \\
\hline 2 Jul. 1989 & $2.2 \mathrm{~m}$ & Cassegrain & RCA\#11 & 30.0 & $512 \times 320$ & 0.35 & $3.0 \times 1.9$ & 0.08 \\
\hline 3 Jul. 1989 & $2.2 \mathrm{~m}$ & Cassegrain & RCA\#11 & 30.0 & $512 \times 320$ & 0.35 & $3 ! 0 \times 1{ }^{\prime} 9$ & 0.01 \\
\hline 4 Jul. 1989 & $2.2 \mathrm{~m}$ & Cassegrain & RCA\#11 & 30.0 & $512 \times 320$ & 0.35 & $3.0 \times 1{ }^{\prime} 9$ & no photometric \\
\hline 25 Jan. 1990 & $2.2 \mathrm{~m}$ & Cassegrain & RCA\#11 & 30.0 & $512 \times 320$ & 0.35 & $3^{\prime} .0 \times 1.9$ & 0.01 \\
\hline 26 Jan. 1990 & $2.2 \mathrm{~m}$ & Cassegrain & $\mathrm{RCA \# 11}$ & 30.0 & $512 \times 320$ & 0.35 & $3^{\prime} .0 \times 1.9$ & 0.02 \\
\hline 27 Jan. 1990 & $2.2 \mathrm{~m}$ & Cassegrain & RCA\#11 & 30.0 & $512 \times 320$ & 0.35 & $3.0 \times 1.9$ & 0.02 \\
\hline 28 Jan. 1990 & $2.2 \mathrm{~m}$ & Cassegrain & RCA\#11 & 30.0 & $512 \times 320$ & 0.35 & $3^{\prime} .0 \times 1.9$ & no photometric \\
\hline 29 Jan. 1990 & $2.2 \mathrm{~m}$ & Cassegrain & RCA\#11 & 30.0 & $512 \times 320$ & 0.35 & $3.0 \times 1{ }^{\prime} .9$ & 0.02 \\
\hline 18 Jun. 1990 & $3.5 \mathrm{~m}$ & Prime & RCA\#10 & 30.0 & $512 \times 320$ & 0.50 & $4.3 \times 2.7$ & 0.08 \\
\hline 19 Jun. 1990 & $3.5 \mathrm{~m}$ & Prime & RCA\#10 & 30.0 & $512 \times 320$ & 0.50 & $4^{\prime} .3 \times 2.7$ & 0.08 \\
\hline 20 Jun. 1990 & $3.5 \mathrm{~m}$ & Prime & RCA\#10 & 30.0 & $512 \times 320$ & 0.50 & $4.3 \times 2.7$ & 0.07 \\
\hline 11 Sep. 1990 & $2.2 \mathrm{~m}$ & Cassegrain & RCA\#11 & 30.0 & $512 \times 320$ & 0.35 & $3^{\prime} .0 \times 1{ }^{\prime} .9$ & no photometric \\
\hline 12 Sep. 1990 & $2.2 \mathrm{~m}$ & Cassegrain & RCA\#11 & 30.0 & $512 \times 320$ & 0.35 & $3.0 \times 1.9$ & 0.08 \\
\hline 13 Sep. 1990 & $2.2 \mathrm{~m}$ & Cassegrain & RCA\#11 & 30.0 & $512 \times 320$ & 0.35 & $3 ! 0 \times 1{ }^{\prime} \cdot 9$ & 0.05 \\
\hline 14 Sep. 1990 & $2.2 \mathrm{~m}$ & Cassegrain & $\mathrm{RCA \# 11}$ & 30.0 & $512 \times 320$ & 0.35 & $3.0 \times 1.9$ & no photometric \\
\hline 11 Dec. 1990 & $2.2 \mathrm{~m}$ & Cassegrain & RCA\#11 & 30.0 & $512 \times 320$ & 0.35 & $3: 0 \times 1.9$ & 0.07 \\
\hline 12 Dec. 1990 & $2.2 \mathrm{~m}$ & Cassegrain & $\mathrm{RCA \# 11}$ & 30.0 & $512 \times 320$ & 0.35 & $3 ! 0 \times 1.9$ & 0.07 \\
\hline 13 Dec. 1990 & $2.2 \mathrm{~m}$ & Cassegrain & $\mathrm{RCA \# 11}$ & 30.0 & $512 \times 320$ & 0.35 & $3^{\prime} .0 \times 1.9$ & 0.07 \\
\hline 14 Dec. 1990 & $2.2 \mathrm{~m}$ & Cassegrain & RCA\#11 & 30.0 & $512 \times 320$ & 0.35 & $3^{\prime} .0 \times 1.9$ & 0.07 \\
\hline 5 Jul. 1991 & $2.2 \mathrm{~m}$ & Cassegrain & GEC\#15 & 22.5 & $1155 \times 768$ & 0.26 & $5 ! 0 \times 3: 3$ & 0.05 \\
\hline 6 Jul. 1991 & $2.2 \mathrm{~m}$ & Cassegrain & GEC\#15 & 22.5 & $1155 \times 768$ & 0.26 & $5.0 \times 3.3$ & no photometric \\
\hline 7 Jul. 1991 & $2.2 \mathrm{~m}$ & Cassegrain & GEC\#15 & 22.5 & $1155 \times 768$ & 0.26 & $5.0 \times 3.3$ & 0.05 \\
\hline 8 Jul. 1991 & $2.2 \mathrm{~m}$ & Cassegrain & GEC\#15 & 22.5 & $1155 \times 768$ & 0.26 & $5^{\prime} .0 \times 3.3$ & 0.06 \\
\hline 9 Jul. 1991 & $2.2 \mathrm{~m}$ & Cassegrain & GEC\#15 & 22.5 & $1155 \times 768$ & 0.26 & $5 ! 0 \times 3 ! 3$ & 0.05 \\
\hline 30 Jan. 1992 & $2.2 \mathrm{~m}$ & Cassegrain & GEC\#15 & 22.5 & $1155 \times 768$ & 0.26 & $5.0 \times 3 ! 3$ & 0.01 \\
\hline 31 Jan. 1992 & $2.2 \mathrm{~m}$ & Cassegrain & GEC\#15 & 22.5 & $1155 \times 768$ & 0.26 & $5.0 \times 3 ! 3$ & 0.01 \\
\hline 1 Feb. 1992 & $2.2 \mathrm{~m}$ & Cassegrain & GEC\#15 & 22.5 & $1155 \times 768$ & 0.26 & $5 ! 0 \times 3 ! 3$ & 0.01 \\
\hline 2 Feb. 1992 & $2.2 \mathrm{~m}$ & Cassegrain & GEC\#15 & 22.5 & $1155 \times 768$ & 0.26 & $5.0 \times 3.3$ & 0.01 \\
\hline
\end{tabular}

i) We first represent the surface brightness profile $\mu$ versus the $r^{1 / 4}$ distance scale (in this graph, the bulge dominated range of the profile, outside the inner region most influenced by the seeing effect, follows a straight line). ii) In this representation, we fit a least squares straight line to the range of the profile dominated by the $r^{1 / 4}$ law. In this way, an initial estimation of the bulge component is obtained. iii) We extrapolate this straight line to the inner part of the profile to roughly correct for the point spread function (PSF) flattening effect in the luminosity profile. iv) We represent, then, the reconstructed profile versus the radius (in this graph the disk dominated range of the profile follows a straight line) and obtain an initial estimate of the disk component fitting by a least squares straight line to the range of the outer profile dominated by the exponential law. v) From this initial guesses we start a $\chi^{2}$ minimization allowing the four parameters to vary freely, within certain limits of the initial values. Finally, we obtain the four basic parameters for the bulge+disk decomposition $\left(\mu_{\mathrm{e}}, r_{\mathrm{e}}, \mu_{0}, d_{\mathrm{L}}\right)$ and the bulge-to-disk ratio, as calculated from the equation:

$$
\frac{B}{D}=3.607\left(\frac{r_{\mathrm{e}}}{d_{\mathrm{L}}}\right)^{2} \operatorname{dex}\left(-0.4\left(\mu_{\mathrm{e}}-\mu_{0}\right)\right)
$$

\subsection{Reliability of our bulge+disk $(B+D)$ decomposition method}

To check the goodness and uncertainty of our $B+D$ decomposition method, we have created a set of 24 bidimensional synthetic images by combining the $r^{1 / 4}$ and exponential laws with the four basic parameters $\left(\mu_{\mathrm{e}}, r_{\mathrm{e}}, \mu_{0}, d_{\mathrm{L}}\right)$, chosen to simulate a wide range of conditions and $B / D$ 
ratios $(B / D=0.01-15.00)$. Each imagewas convolved with a gaussian PSF (with a typical seeing of FWHM = $2^{\prime \prime}$ ) and an artificial noise image was added. From these images, we used the PROF routine to obtain the elliptical contours and the radial surface brightness profiles. Then we applied our $B+D$ decomposition procedure to see how well the theoretical input values of the four parameters and the $B / D$ ratio were reproduced.

The obtained results and the estimated uncertainty in each parameter show that, in general, the disk parameters are better reproduced than the bulge parameters because the latter are worse defined, being more influenced by the seeing correction. In addition, the highest errors are observed in the cases in which one of the components (bulge or disk) clearly dominates over the other one. This confirms the results of Kent (1985b): a $B+D$ decomposition should only be considered reliable when $B / D<1.7$. On the average, for the complete set of models, the rms scatters (fitted - theoretical) are $\sim 0.09$ and $0.08 \mathrm{mag} / \mathrm{arcsec}^{2}$ for the $\mu_{\mathrm{e}}, \mu_{0}$ surface brightnesses, respectively and corresponds to $7 \%$ and $3 \%$ errors for the $r_{\mathrm{e}}, d_{\mathrm{L}}$ scales, respectively. For the cases with a more realistic $B+D$ decomposition $(B / D<1.7)$, these errors are decreased to $\sim 0.03 \mathrm{mag} / \operatorname{arcsec}^{2}$ for $\mu_{\mathrm{e}}, \mu_{0}$ and $4 \%, 2 \%$ for $r_{\mathrm{e}}, d_{\mathrm{L}}$, respectively. The rms scatter of the global fits is typically $\sigma \sim 0.03 \mathrm{mag} / \operatorname{arcsec}^{2}$.

Table 3 lists the results of the $B+D$ decomposition for our sample (corrected for inclination using the angle derived from the ellipticity $\epsilon$, averaged for the best defined five outermost isophotes, and corrected for galactic extinction) together with the associated errors, and the rms scatter of the global fit for the UCM objects.

\subsection{Concentration indices and mean photometric parameters}

The use of the $B+D$ decomposition to characterize the radial distribution of light in the galaxies presents some inherent problems. On the one hand, the empirical laws used for the $B+D$ fit are just first approximations. On the other hand, the PSF effect can seriously affect the results of this decomposition. Therefore, additional criteria (concentration indices and mean surface brightnesses), independent of the decomposition procedure, have been also used to perform the morphological classification of the sample.

Since the first works by de Vaucouleurs \& Agüero (1973) and Fraser (1977), concentration indices (ratio between two radii including given fractions of the total luminosity of the galaxy) have been widely used as morphological indicators, mainly by de Vaucouleurs (1977); Okamura et al. (1984); Kent (1985b); Gavazzi et al. (1990) and Doi et al. (1993). In this work, we have computed for all the UCM objects, by integration in the radial profiles, the $c_{31}$ concentration index introduced by de Vaucouleurs (1977) and the $c_{42}$ index of Kent (1985b). We have also calcu- lated the major $\left(2 a_{24}\right)$ and minor $\left(2 b_{24}\right)$ axes at the 24 $\mathrm{mag} / \operatorname{arcsec}^{2}$ isophote, the equivalent radii of the 24 and $24.5 \mathrm{mag} / \operatorname{arcsec}^{2}$ isophotes $\left(r_{24}\right.$ and $\left.r_{24.5}\right)$, the apparent magnitudes inside these isophotes $\left(m_{24}\right.$ and $\left.m_{24.5}\right)$, the effective radius $\left(a_{\mathrm{e}}\right)$, the mean surface brightness inside the effective radius $\left(\bar{\mu}_{\mathrm{e}}\right)$, and the mean surface brightness inside the $24.5 \mathrm{mag} / \operatorname{arcsec}^{2}$ radius $\left(\bar{\mu}_{24.5}\right)$; both brightnesses were then corrected for galactic extinction. Finally, we have computed the concentration index $c_{\text {in }}(\alpha)$ with $\alpha$ $=0.3$, introduced by Doi et al. (1993) due to its utility to segregate among morphological types when it is jointly used with the mean surface brightness $\bar{\mu}_{24.5}$. All these photometric parameters are listed in Tables 4 and 5 . In order to estimate the uncertainties in these parameters, a set of theoretical model profiles was constructed. The application of our integration method gives typical errors for the concentration indices from $2 \%$, for the pure exponential profile models, to $10 \%$, for the pure $r^{1 / 4}$ models, and from $1 \%$, in exponential models, to $4 \%$, in the $r^{1 / 4}$ models, for the photometric radii. Since most of the UCM galaxies are disk dominated systems, the actual errors are close to the lower limits of the above uncertainties. Finally, estimated errors for the mean surface brightnesses are typically of $0.1 \mathrm{mag} / \operatorname{arcsec}^{2}$.

\section{Morphological interpretation of the photome- tric results}

One of the fundamental objectives of any photometric study of galaxies is to achieve a reliable morphological classification. In our case, this is a difficult task due to the small angular size of the objects in the UCM sample. Moreover any classification based only on their appearance on the direct image is always doubtful. In this work, we have jointly used both the information from the visual inspection of the images (appearance of the arms, signs of interaction or mergers, etc.) and the interpretation of the surface brightness profile obtained for each object, by means of its $B+D$ decomposition and some photometric parameters (concentration indices and mean surface brightnesses, independent of any decomposition method).

Therefore, with the aim of assigning a Hubble type to each object of the UCM sample, we have used five different criteria from several authors, previously tested in large samples of galaxies with known Hubble types, all of them based in the correlations found between the photometric parameters and the Hubble type. These relations, and their limitations, are the following:

- The correlation between $B / T$ ratio and the Hubble type, where $B / T$ is the ratio between the bulge and total luminosities of the galaxy $(B / T=B /(B+D))$. This correlation, studied by Kent (1985b) for a sample of bright galaxies, is based on the well known trend of $B / T$ to decrease from early to later type galaxies; so for E-S0 galaxies, the bulge component dominates over the 
disk component, whereas for Sc galaxies the bulge contribution to the total luminosity is only $\sim 10 \%$ (Kent 1985b; Simien \& de Vaucouleurs 1986). However, one must be aware of the limitations of this relation since there is a clear overlap between different Hubble types (Kent 1985b).

- The dependence of the Hubble type of the galaxy on the position in the $c_{\text {in }}(\alpha)$ versus $\bar{\mu}_{24.5}$ plane of Doi et al. (1993), elaborated from a large sample of nearby galaxies and applied to other samples of galaxies with smaller apparent sizes. Studying the position of the galaxies with respect to the segregation line $c_{\text {in }}(\alpha=0.3)$ $=-0.12 \bar{\mu}_{24.5}+3.22$, Doi et al. are able to classify successfully galaxies into early and late types in $85 \%$ of the cases. In spite of the inherent problems associated to this statistical segregation, specially in the Sa contamination zone, the set of graphics elaborated by these authors allows a segregation of galaxies in four Hubble type groups.

- The correlation between the $c_{31}$ concentration index, defined by de Vaucouleurs (1977), and the Hubble type, in the sense that indices of early type galaxies are systematically higher than those of late type objects, as Gavazzi et al. (1990) have also shown. The limitation of this method arises from the intrinsic scatter of the above relation, mainly for early type galaxies.

- The correlation between the $c_{42}$ concentration index, defined by Kent (1985b), and the Hubble type, showing a similar trend and the same statistical problems as the $c_{31}$ index.

- The correlation between the mean surface brightness inside the effective radius, $\vec{\mu}_{\mathrm{e}}$, and the morphological type. As Kent (1985b) has shown, $\bar{\mu}_{\mathrm{e}}$ gets brighter for early types, but the limitations of the method arise from the overlapping between different types together with the small change in the $\bar{\mu}_{\mathrm{e}}$ value from $\mathrm{E}$ to $\mathrm{Sc}$ types.

In this work, galaxies are classified according to the classical Hubble types (E, S0, Sa, ..., Irr) plus the BCD (blue compact dwarf) type (due to the specific interest of these objects in the UCM survey). Since the BCD category usually encompasses objects with a wide variety of properties, in the present work we classify as BCDs those galaxies having all of the following properties: compact appearance in the direct image, linear size $\left(D_{24}\right)$ lower than $10 \mathrm{kpc}$, luminosity $M_{\mathrm{r}}>-19$, and photometric parameters typical of later Hubble types. Moreover, since the true nature as BCD must be spectroscopically confirmed, the spectral information available for these candidates (Gallego et al. 1995) has also been used.

After a detailed analysis of the types given by each criterion, which in a number of cases do not agree, and using the information from the inspection of the direct image, we have assigned a final morphological type to each object of the sample. This final type, taken as the most probable, has been estimated as that in which most criteria agree. Since this procedure is not completely objective, in Table 6 we list for each UCM object the range of Hubble types according to each one of the five criteria, together with the final adopted type. The estimated typical uncertainty in the adopted morphological types is about one Hubble type.

In Table 7 we present individual comments for each object of the UCM sample, including a brief description about the morphological classification. Alternative names for the objects with previous identifications are also given. In Fig. 1 we present, for each UCM object, a gray scale image, a contour map (in which isophotes are plotted in $0.5 \mathrm{mag} / \operatorname{arcsec}^{2}$ intervals) and the observed surface brightness radial profile with the $B+D$ decomposition and the $(B+D)$ fitted and PSF deconvolved profile (solid line).

\section{Summary}

The UCM ELGs survey is being carried out with the main goals of identifying and studying new young, low metallicity galaxies and quantifying the properties of the star formation in the local universe. To achieve these purposes we are developing an intensive effort to characterize photometric and spectroscopically the nature of the selected objects. In this Paper I we have presented the $r$ images, observations and reductions of a sample of ELGs selected from the UCM survey. For a sample of 212 UCM objects we have obtained the basic parameters (size and luminosity) and, via surface photometry techniques, a set of specific photometric parameters (bulge+disk decomposition, concentration indices and mean surface brightnesses) have been computed to characterize the luminosity profiles. Finally, from this set of parameters, and using the known trends between them and the morphology, we have determined a Hubble type for each ELG of the UCM sample.

In a next Paper (Vitores et al. 1995) we will present a statistical analysis of the morphological parameters, both intrinsical to the UCM sample, and extrinsical in comparison with other samples of normal and emission-line galaxies, and we will analyse the possible influence of environment effects in the presence and nature of ELGs.

Acknowledgements. The authors are indebted to J. Gorgas for his continuous cooperation, valuable ideas and careful reading of the manuscript. This work was supported in part by the Spanish Programa Sectorial de Promoción General del Conocimiento under grant PB93-456.

\section{References}

Acker A., Ochsenbein F., Stenholm B., Tylenda R., Marcout J., Schohn C., 1992, The Starsbourg-ESO Catalogue of Galactic Planetary Nebulae, ESO edit. Germany

Barbieri C., Rosino L., 1972, Ap\&SS 16, 324 
Berger J., Cordoni J.P., Fringant A.M., Guibert J., Moreau O., Reboul H., Vanderriest C., 1991, A\&AS 87, 389

Berger J., Fringant A.M., 1977, A\&AS 28, 123

Bothun G.D., Dressler A., 1986, ApJ 301, 57

Bothun G.D., Halpern J.P., Lonsdale C.J., Impey C., Schmitz M., 1989, ApJS 70, 271

Burstein D., Heiles C., 1982, AJ 87, 1165

Cawson M., 1983, Ph. D. thesis, University of Cambridge

de Grijp M.H.K., Miley G.K., Lub J., 1987, A\&AS 70, 95

de Juan y Ruiz L., 1994, Ph. D. thesis, Universidad Autónoma de Madrid

de Vaucouleurs G., 1948, Ann. Ap. 11, 247

de Vaucouleurs G., 1977, Evolution of Galaxies and Stellar Populations. In: Larson R., Tynsley B. (eds.), Yale Univ. Observatory, New Haven, 43

de Vaucouleurs G., Agüero E., 1973, PASP 85, 150

de Vaucouleurs G., de Vaucouleurs A., Corwin H.G. Jr., Buta R.J., Paturel G., Fouqué P., 1991, Third Reference Catalogue of Bright Galaxies. New York: Springer (RC3)

Doi M., Fukugita M., Okamura S., 1993, MNRAS 264, 832

Dressler A., Shectman A., 1988, AJ 95, 284

Freeman K.C., 1970, ApJ 160, 811

Fraser C.W., 1977, A\&AS 29, 161

Gallego J., Zamorano J., Rego M., Vitores A.G., 1994, A\&A 290,705

Gallego J., Zamorano J., Rego M., Alonso O., Vitores A.G., 1995, A\&AS (submitted)

Gavazzi G., Boselli A., Kennicutt R., 1991, AJ 101, 1207

Gavazzi G., Garilli B., Boselli A., 1990, A\&AS 83, 399

Gavazzi G., Randone I., 1994, A\&AS 107, 285

Gavazzi G., Trinchieri G., 1989, ApJ 342, 718

González-Serrano J.I., Pérez-Fournon I., 1991, A\&A 249, 75

Kazaryan M.A., Kazaryan E.S., 1980, Afz 16, 17

Kent S.M., 1985a, PASP 97, 165
Kent S.M., 1985b, ApJS 59, 115

MacAlpine G.M., Smith S.B., Lewis D.W., 1977a, ApJS 34, 95

MacAlpine G.M., Lewis D.W., Smith S.B., 1977b, ApJS 35, 203

Mazzarella J.M., Balzano V.A., 1986, ApJS 62, 751

Okamura S., Kodaira K., Watanabe M., 1984, ApJ 280, 7

Osterbrock D.E., Cohen R.D., 1982, ApJ 261, 64

Paturel G., Fouqué P., Bottinelli L., Gouguengheim L., 1989, Catalogue of Principal Galaxies (PGC), Observatoires de Lyon et Paris-Meudon, Lyon (France)

Rego M., Zamorano J., Gallego J., Vitores A.G., 1994, A\&A 281,348

Salzer J.J., MacAlpine G.M., Boroson T.A., 1989, ApJS 70, 447

Sanduleak N., Pesch P., 1990, ApJS 72, 291

Schombert J.M., Bothun G.D., 1987, AJ 93, 60

Simien F., de Vaucouleurs G., 1986, ApJ 302, 564

Stephenson C.B., Pesch P., MacConnell D.J., 1992, ApJS 82, 471

Takase B., Miyauchi-Isobe N., 1993, Publ. Natl. Obs. Japan 3, 169

Thuan T.X., Gunn J.E., 1976, PASP 88, 543

van Haarlem M.P., Cayón L., Gutiérrez de la Cruz C., Martínez-González E., Rebolo R., 1993, MNRAS 264, 71

Vitores, A. G., Zamorano, J., Rego, M., Gallego, J., \& Alonso, O. 1996, A\&AS (accepted) (Paper II)

Weedman D.W., 1978, MNRAS 184, 11P

Zamorano J., Gallego J., Rego M., Vitores A.G., GonzálezRiestra R., 1992, AJ 104, 1000

Zamorano J., Rego M., Gallego J., Vitores A.G., GonzálezRiestra R., Rodríguez-Caderot G., 1994, ApJS 95, 387

Zamorano J., Gallego J., Rego M., Vitores A.G., Alonso O., 1995, ApJS (submitted) 

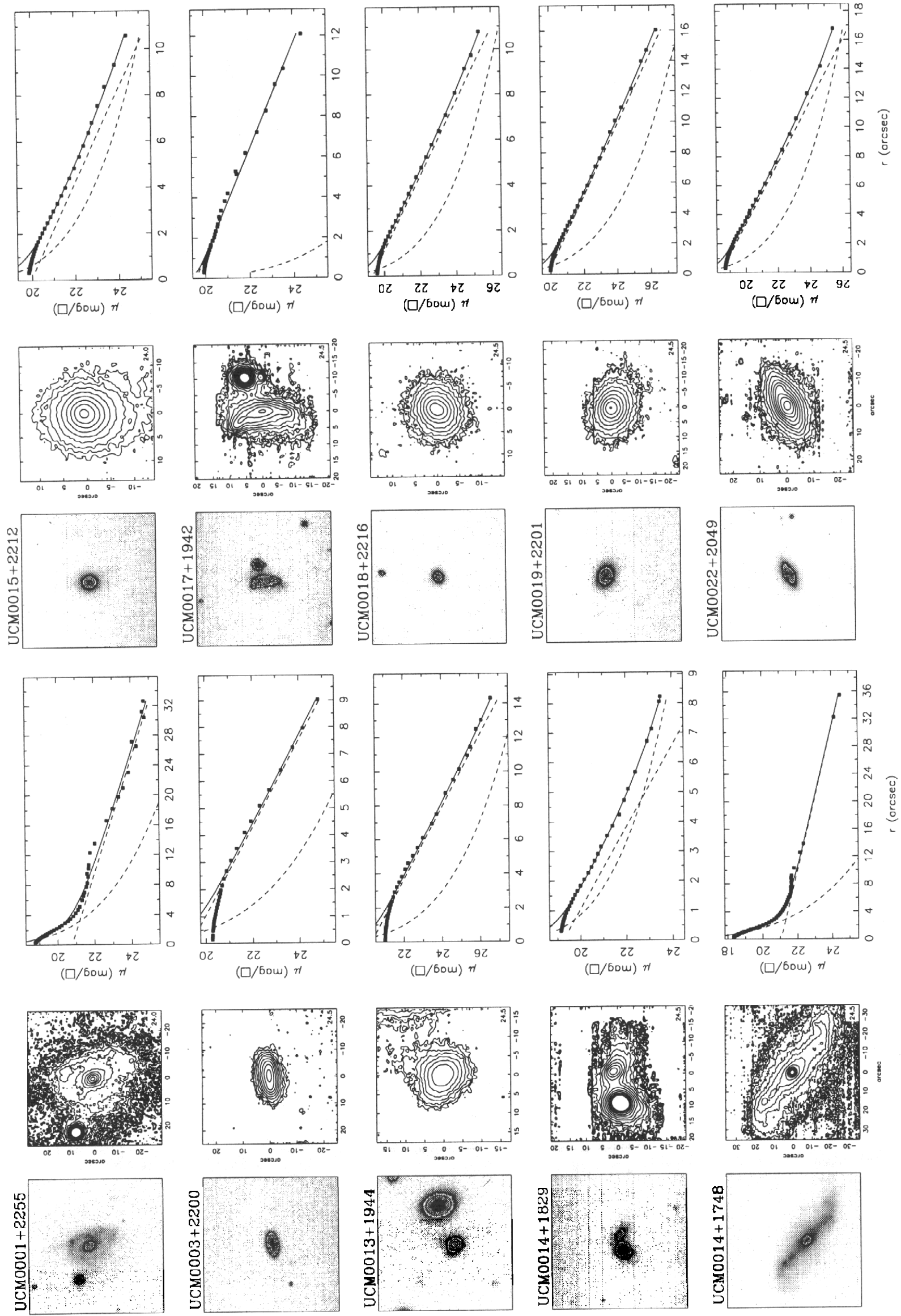

Fig. 1. Gray-scale maps, contour plots (the number at the lower right corner gives the faintest isophote represented) and brightness profiles for the UCM objects. North is up and east is to the left. (See text for details) 

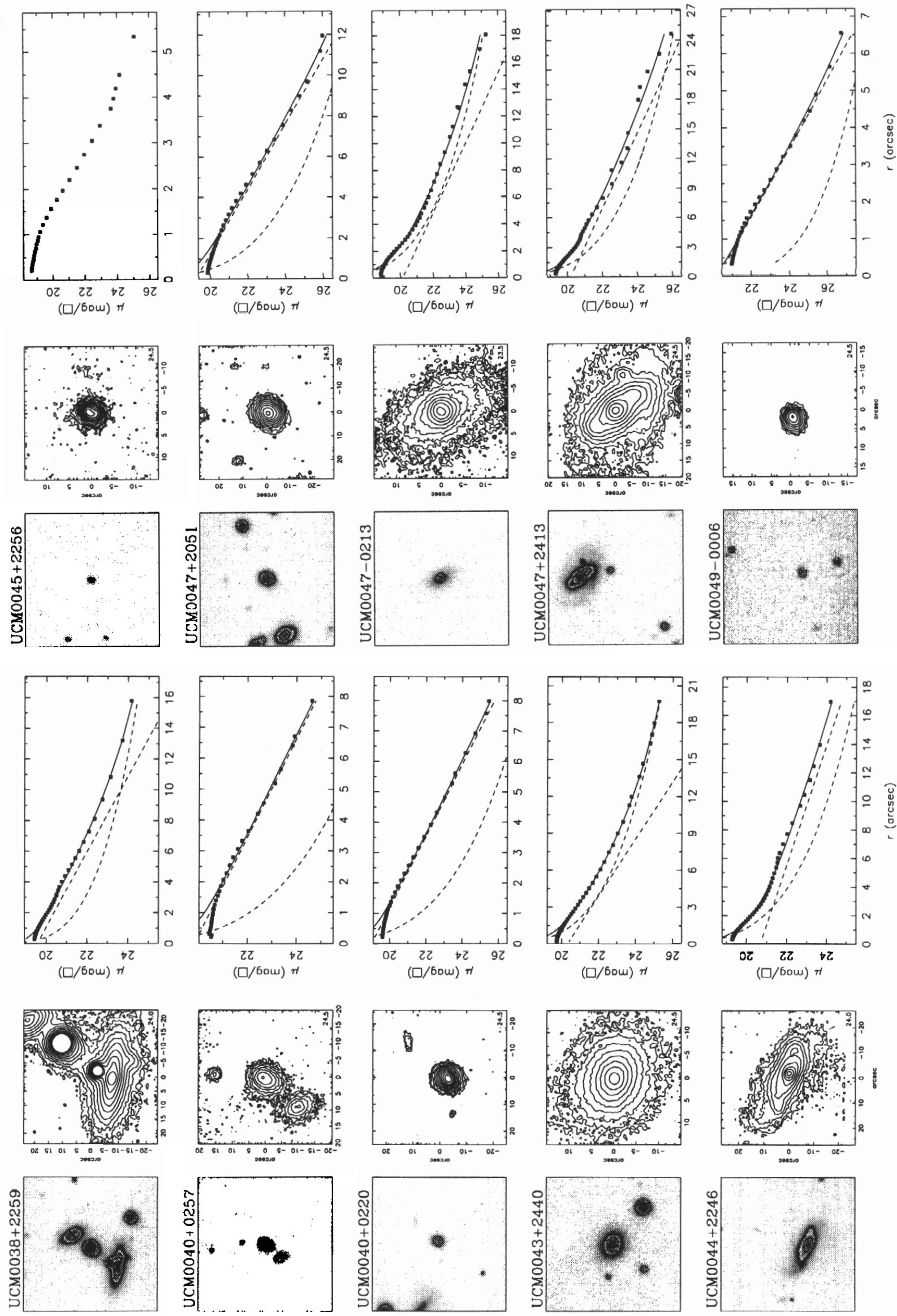

Fig. 1. continued 

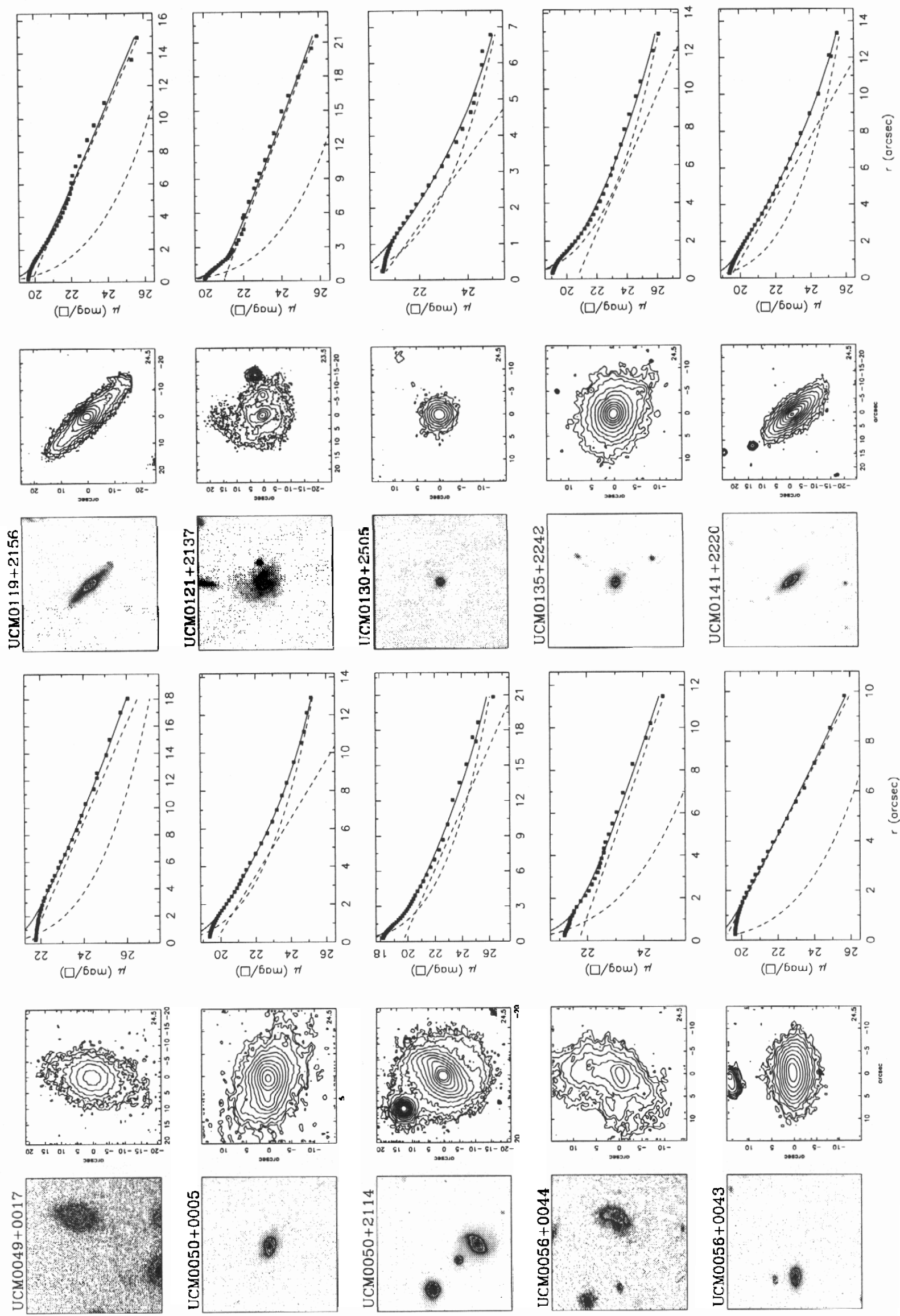

Fig. 1. continued 

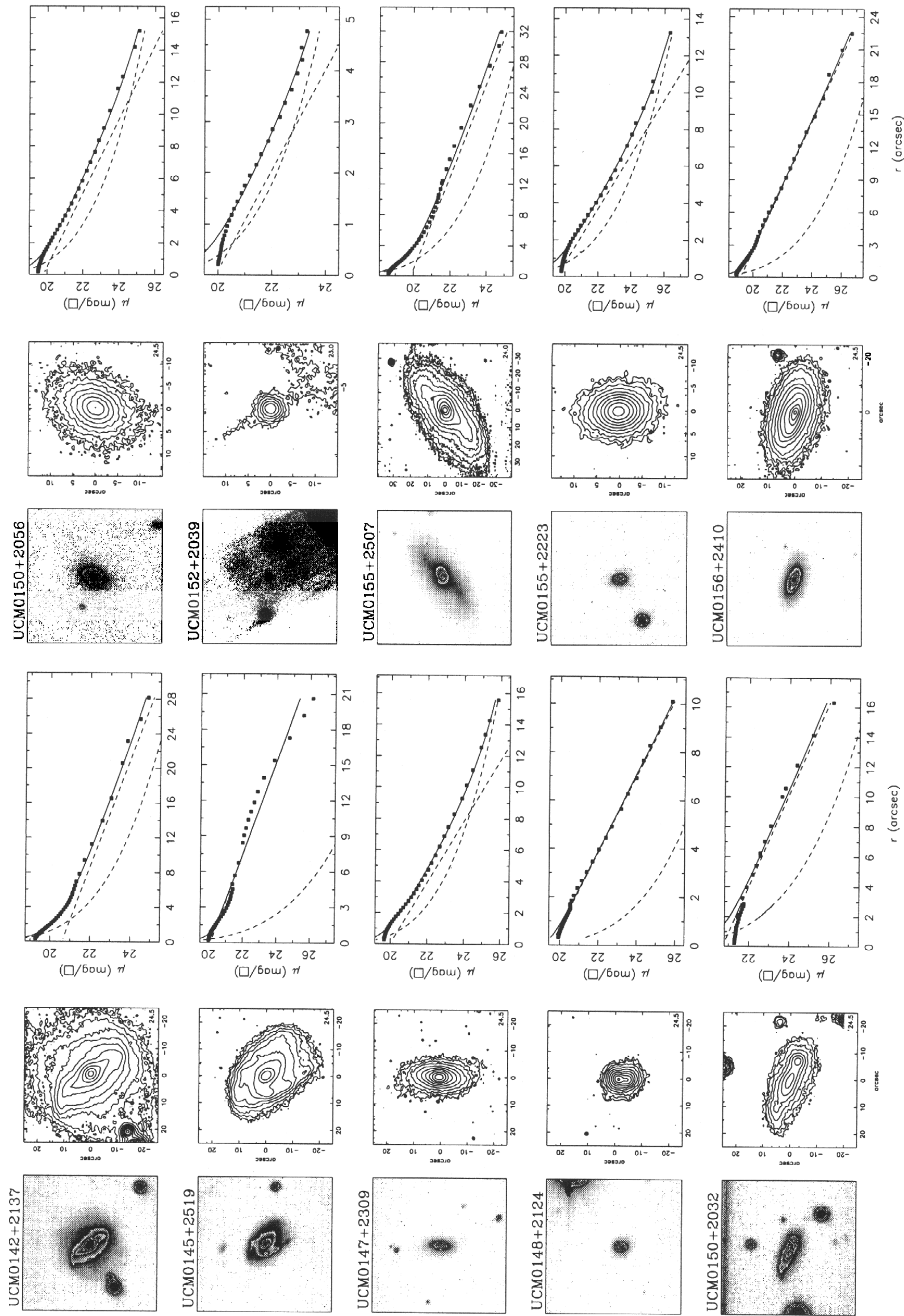

Fig. 1. continued 

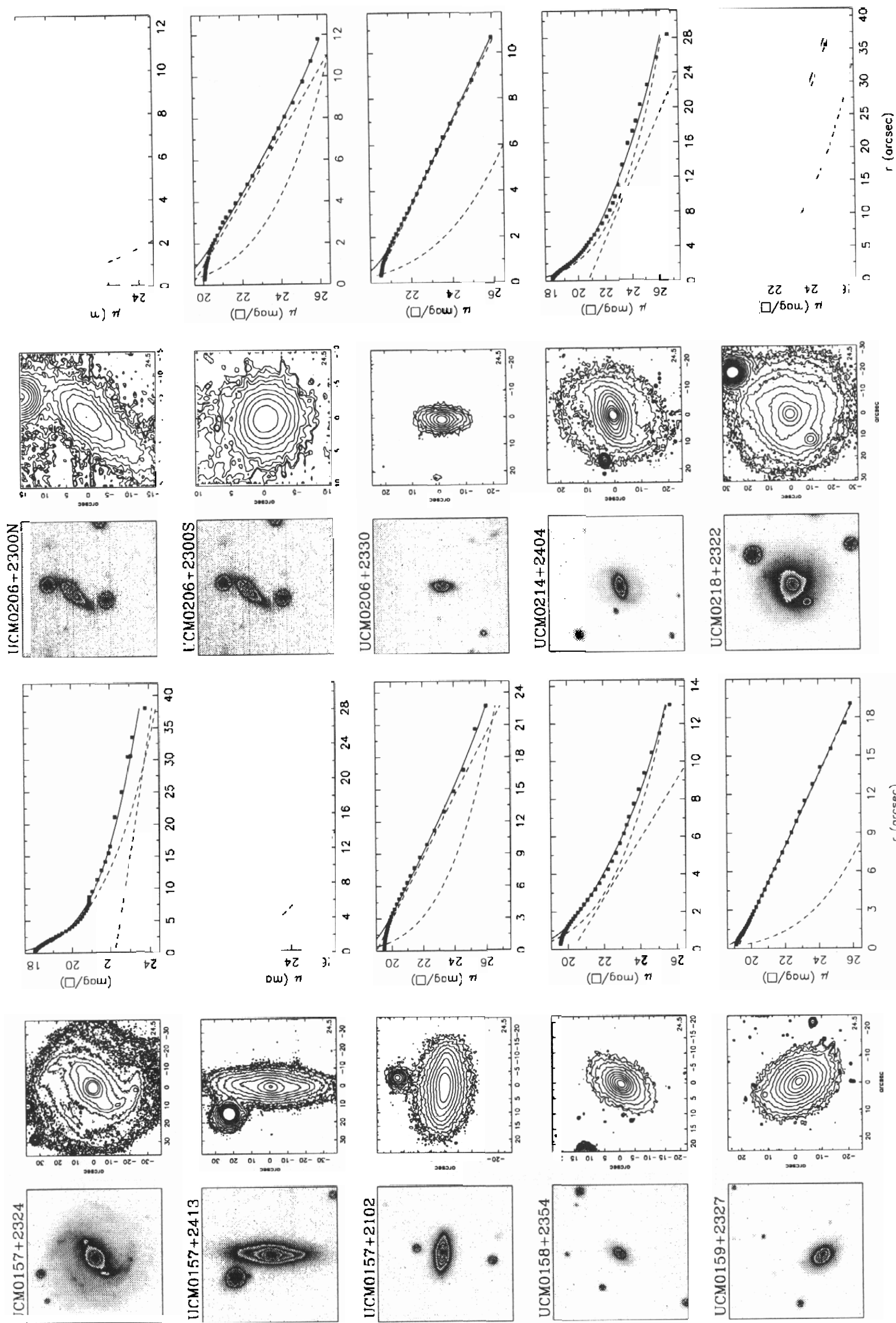

Fig. 1. continued 

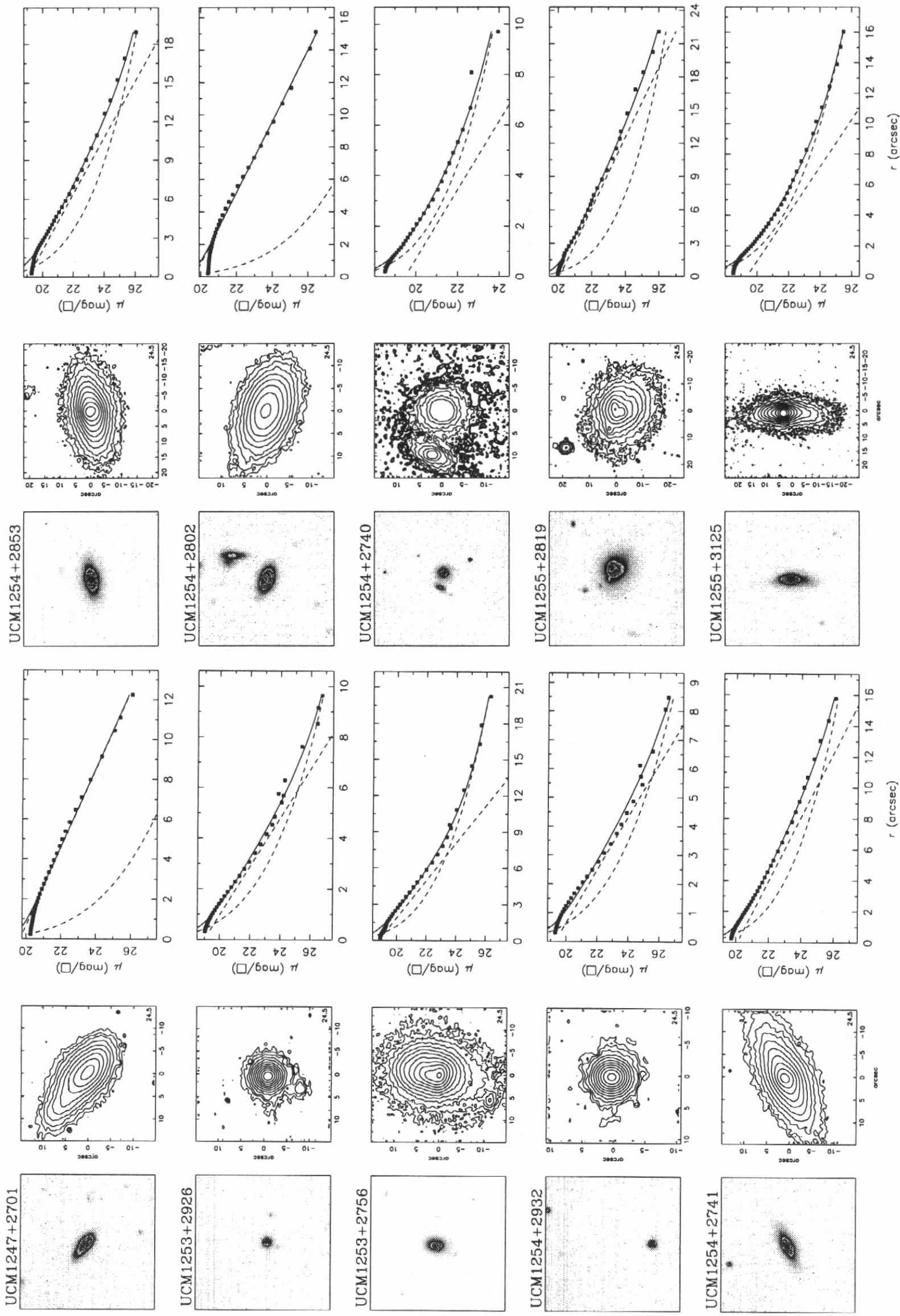

Fig. 1. continued 

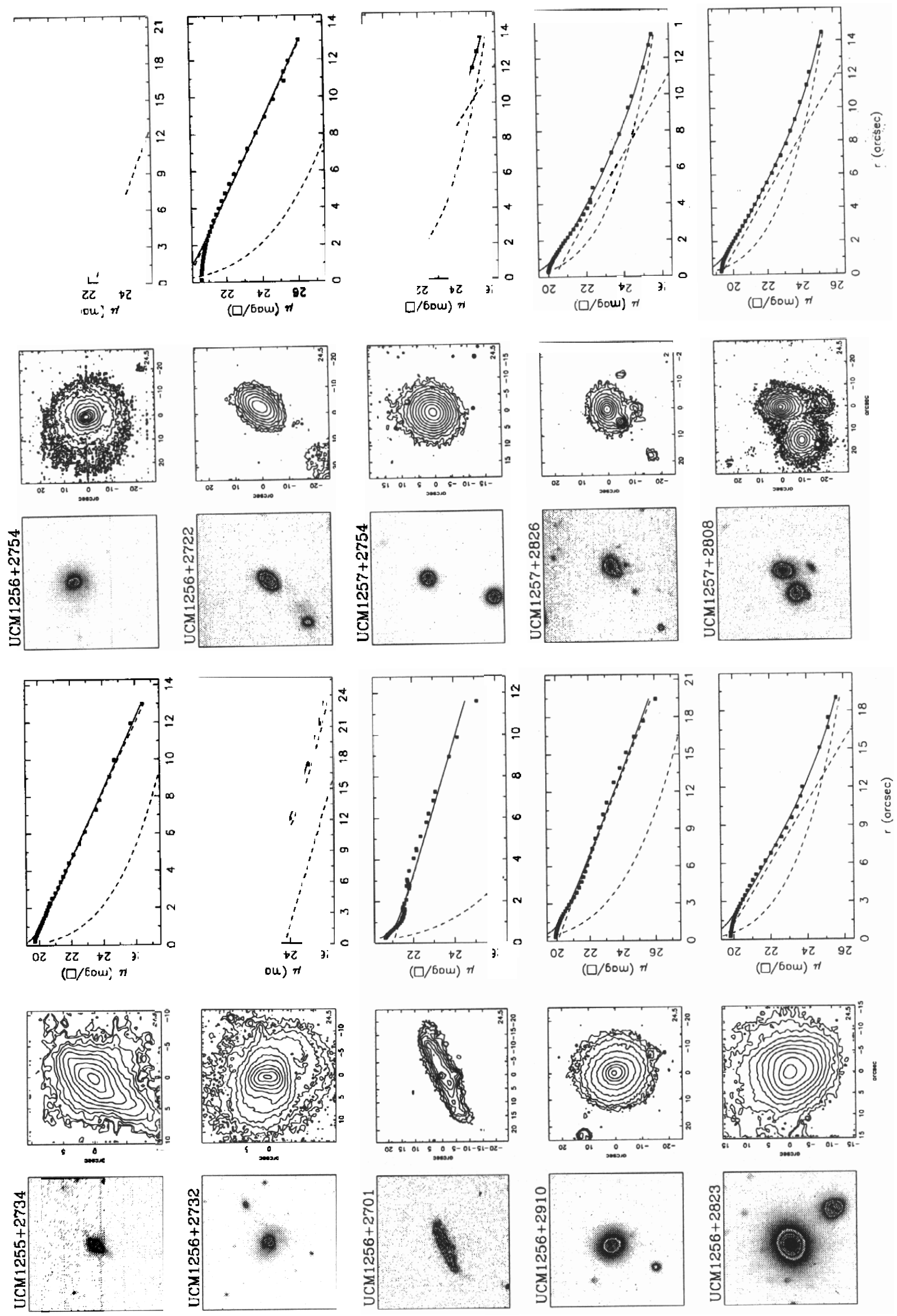

Fig. 1. continued 

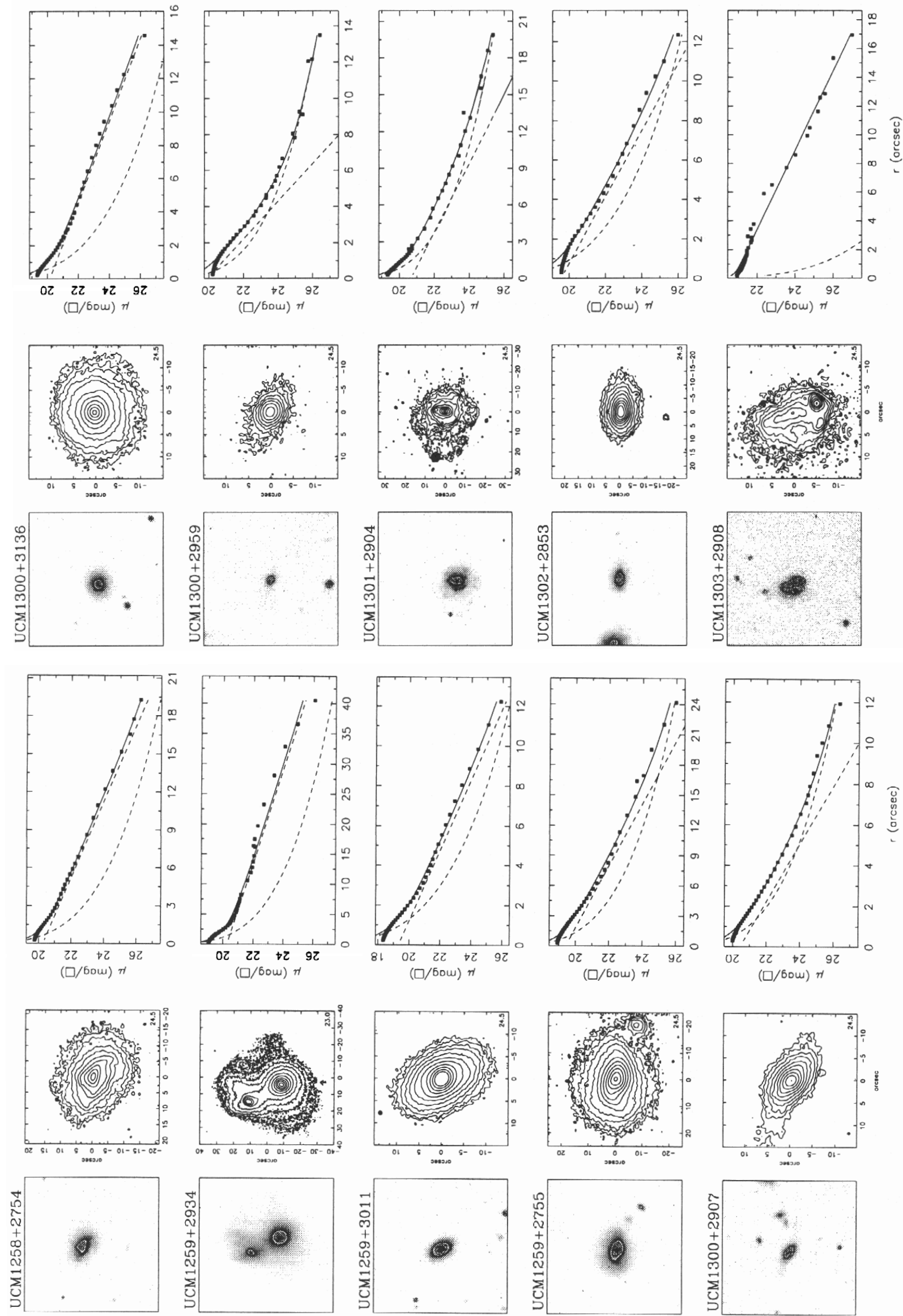

Fig. 1. continued 

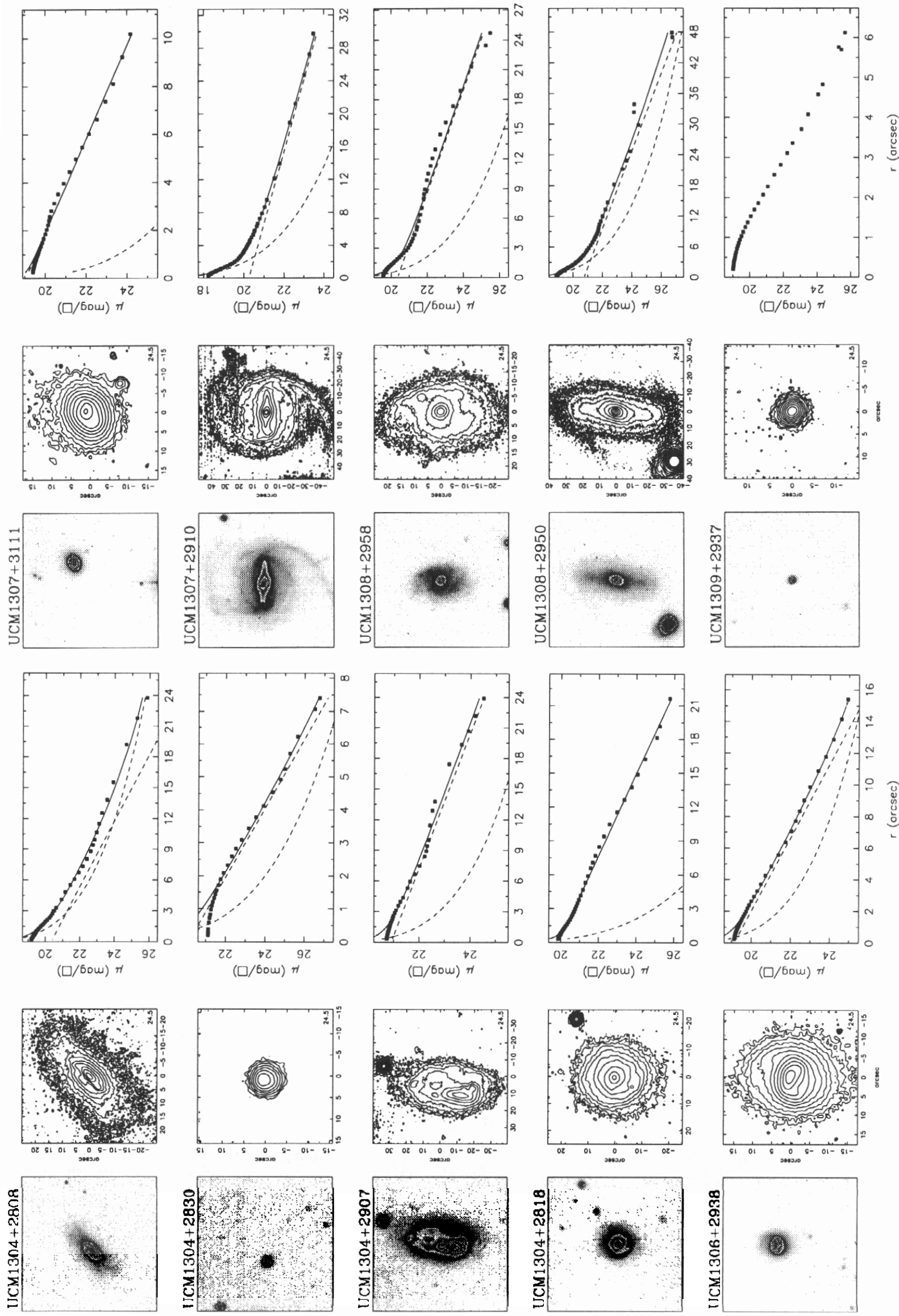

Fig. 1. continued 

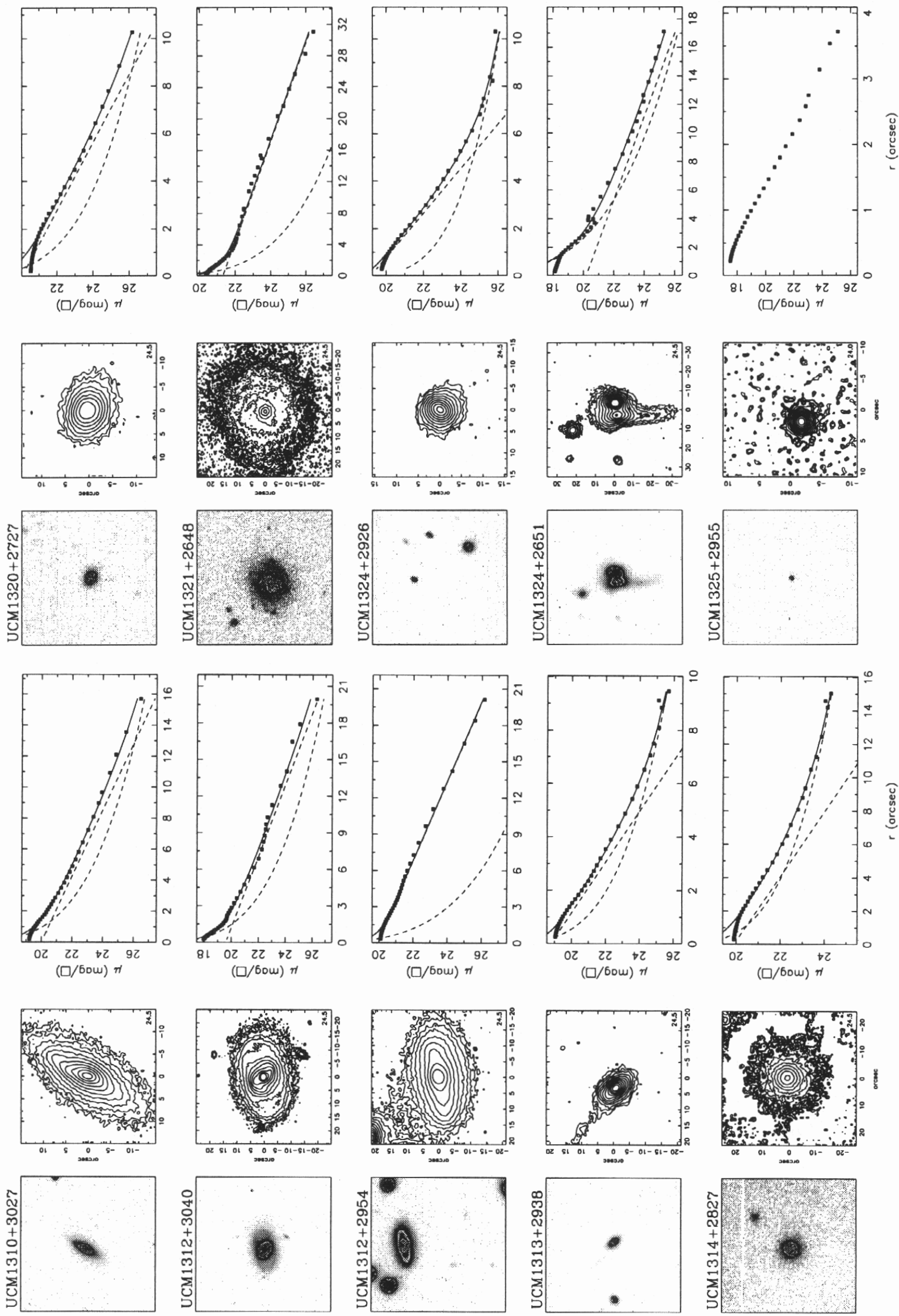

Fig. 1. continued 

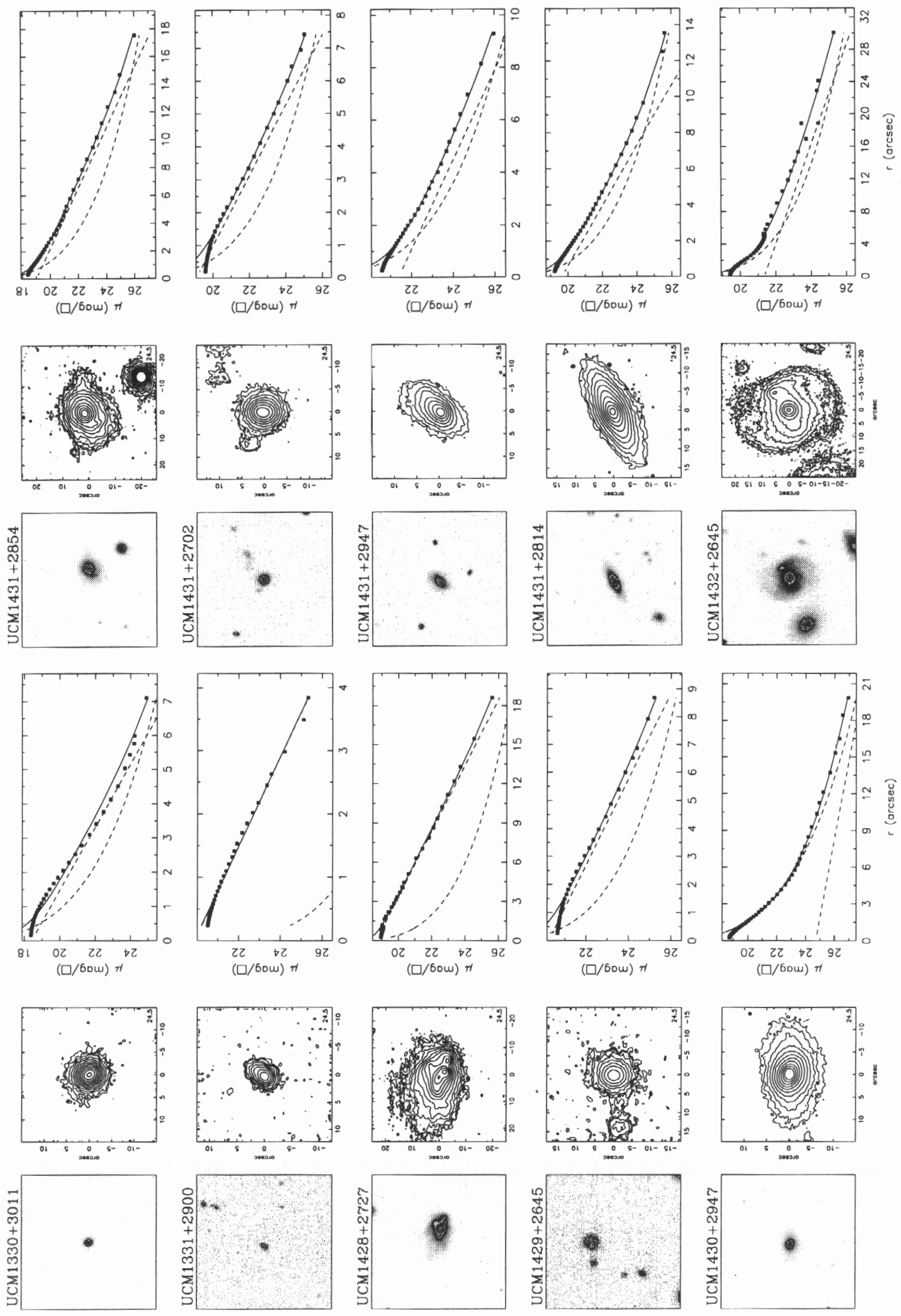

Fig. 1. continued 

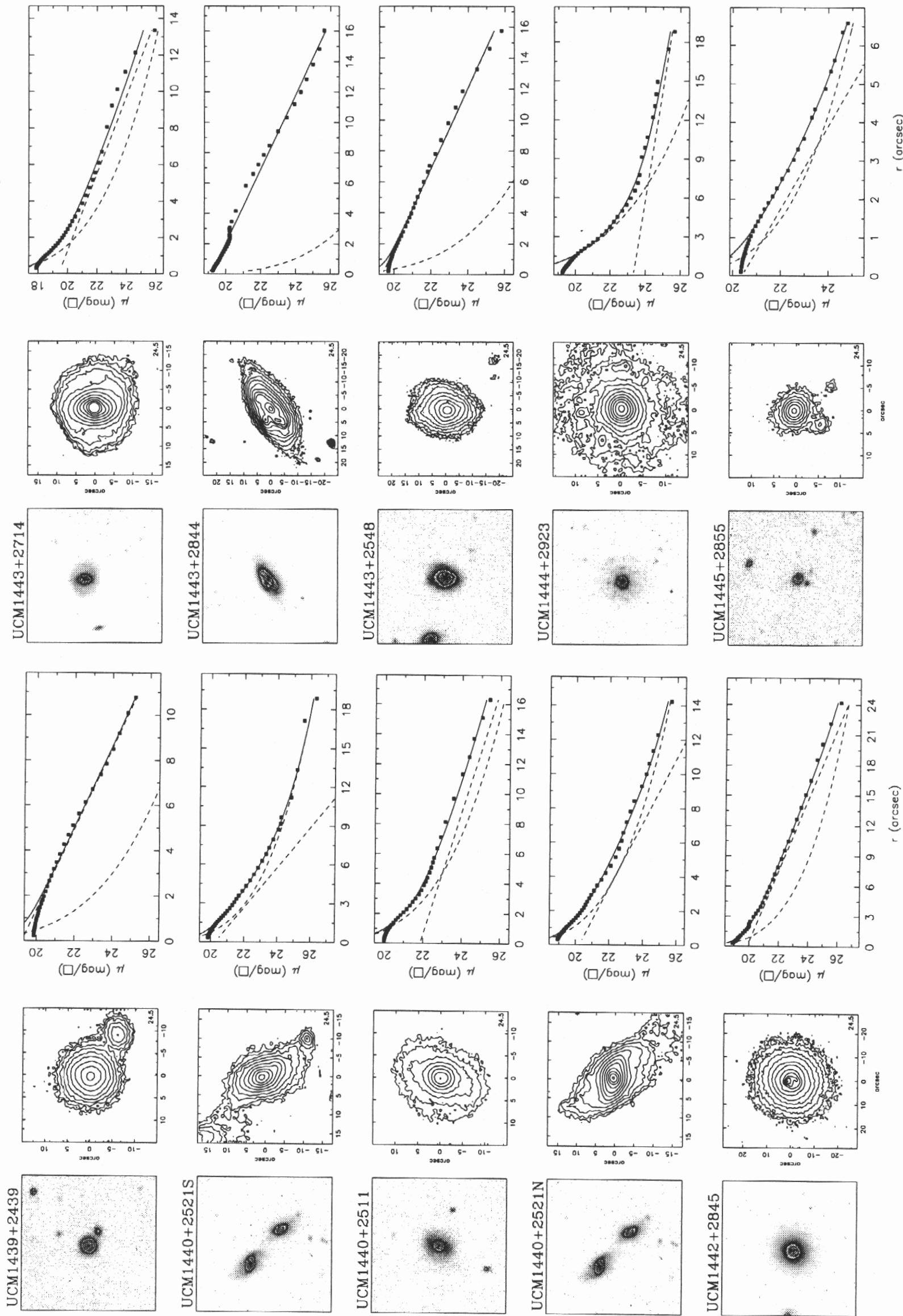

Fig. 1. continued 


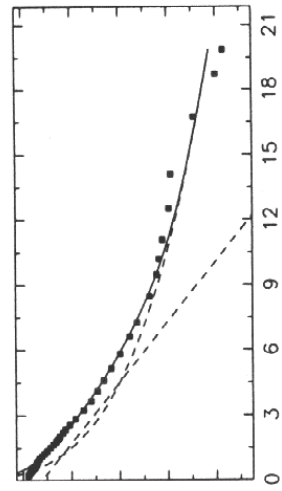

$81 \quad 02 \quad 22 \quad 02 \quad 92$ $(\square / 60 w) \pi$
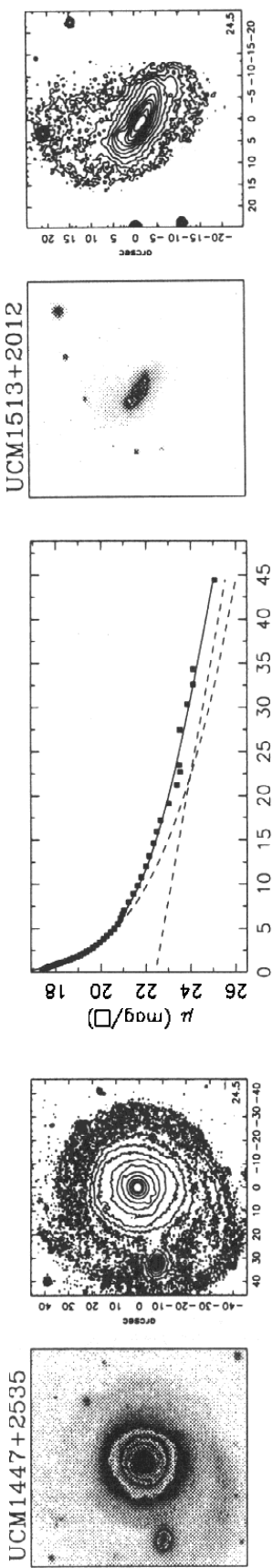

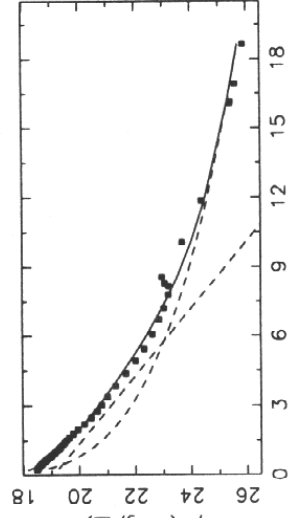

(口/6ow) $\pi$
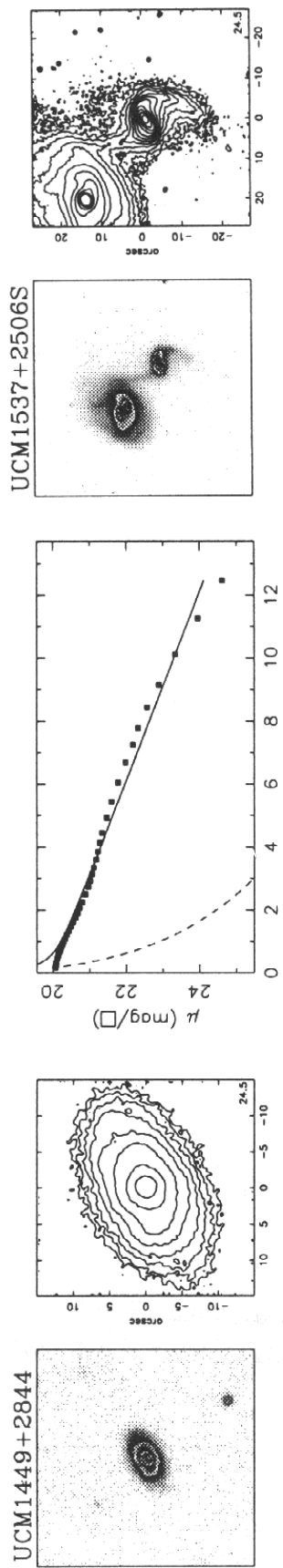

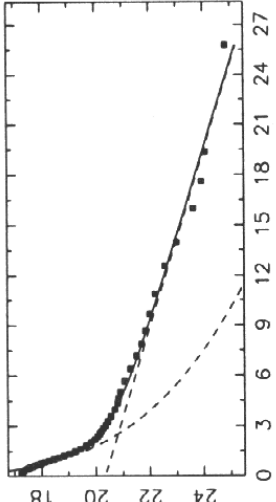

$(\square / 60 w) n$
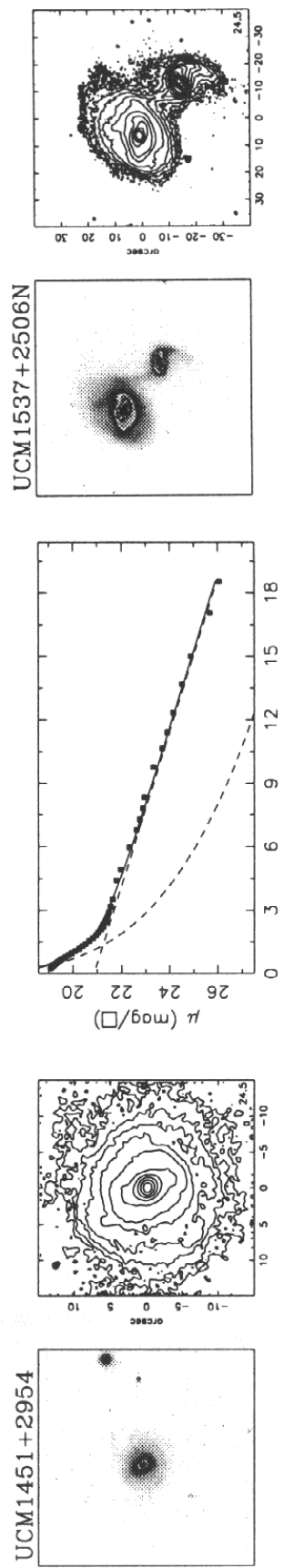

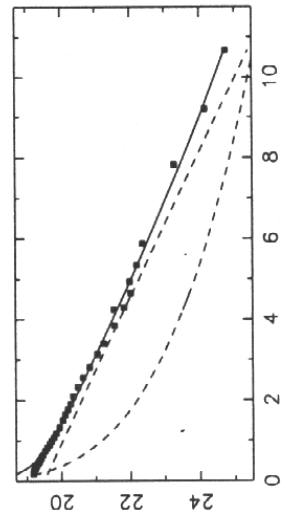

$(\square / 60 w) \pi$
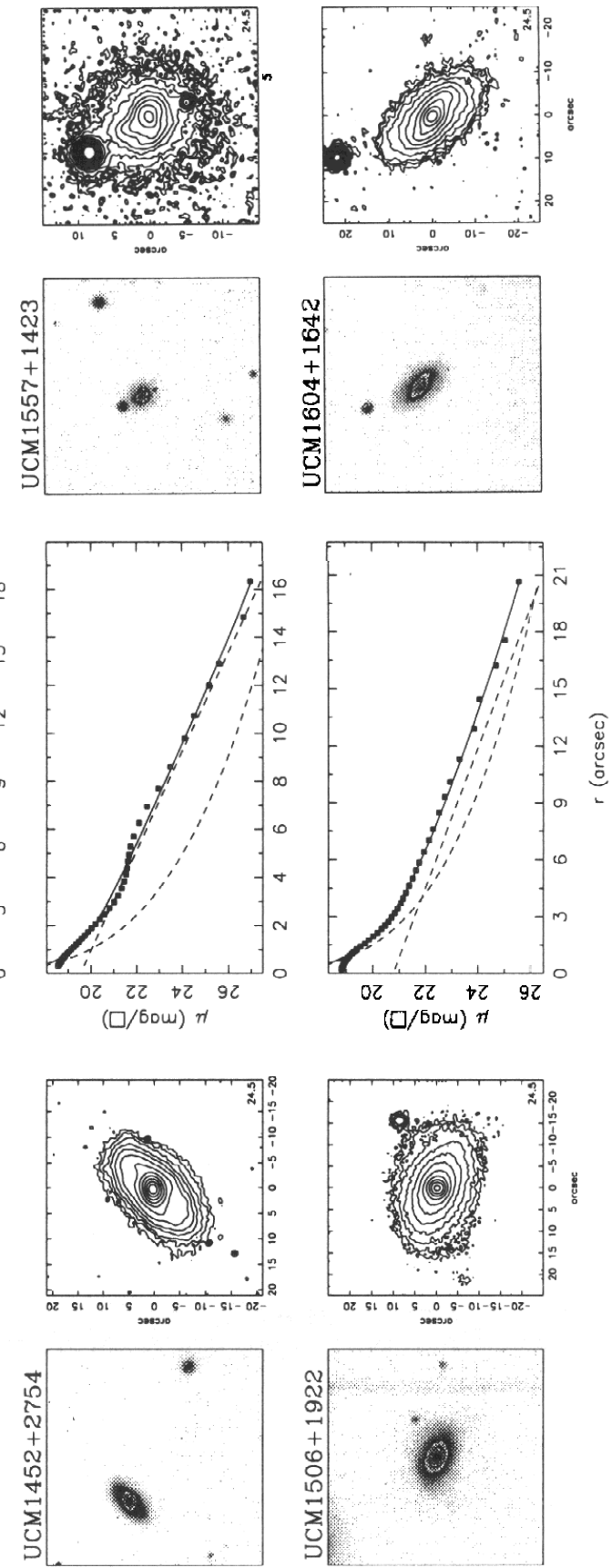

Fig. 1. continued 

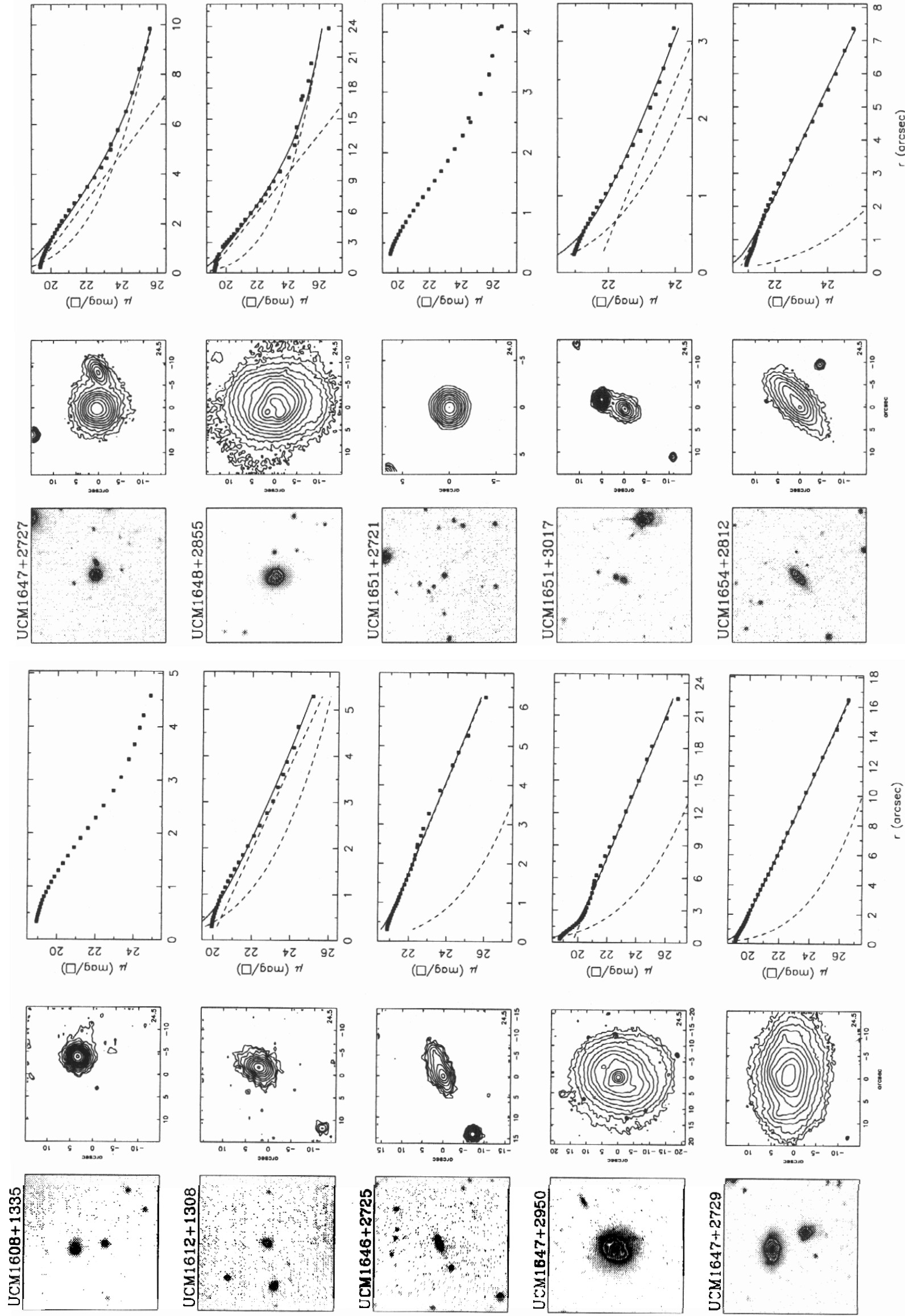

Fig. 1. continued 

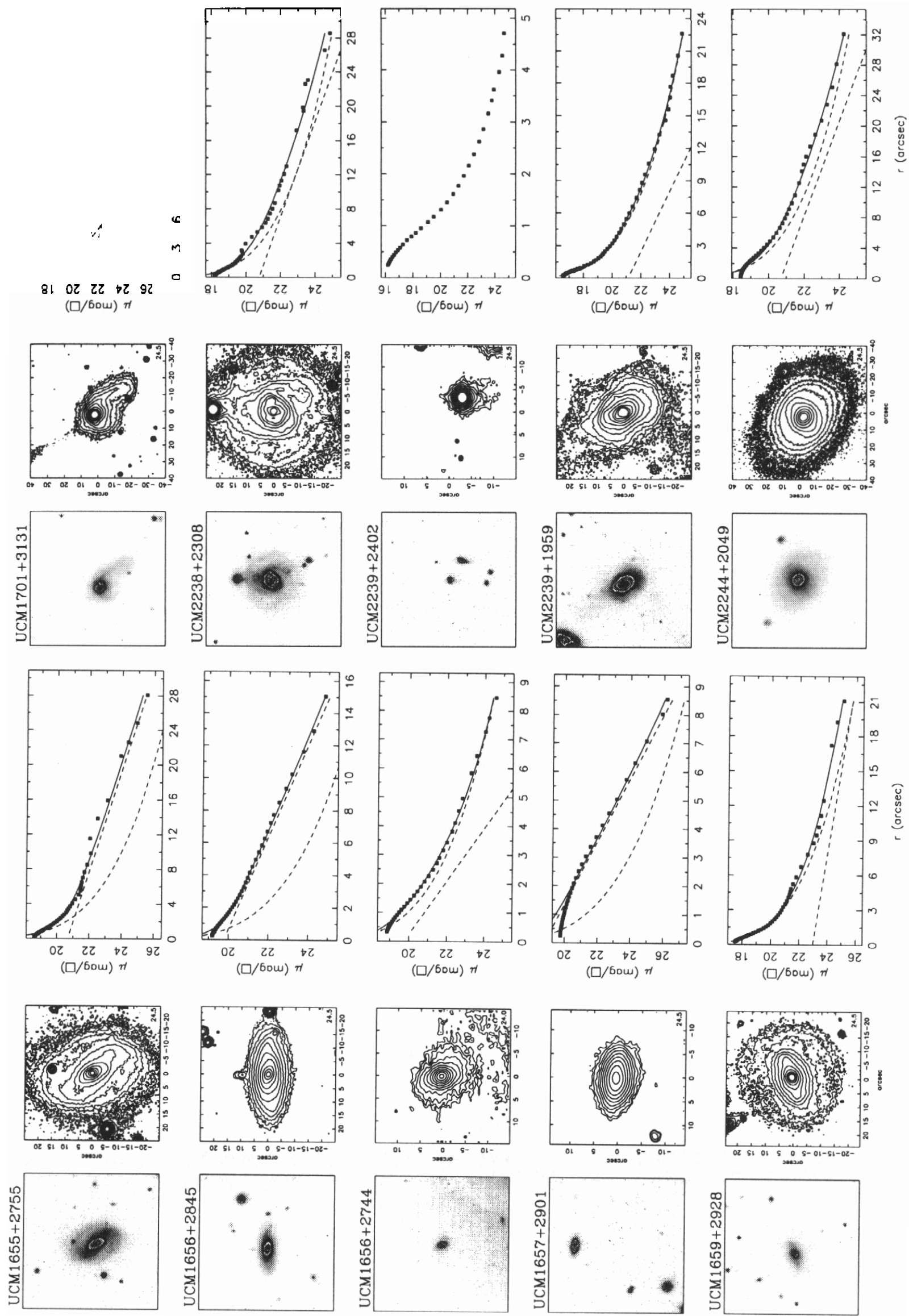

Fig. 1. continued 
A.G. Vitores et al.: Photometric and morphological analysis of UCM galaxies. I.
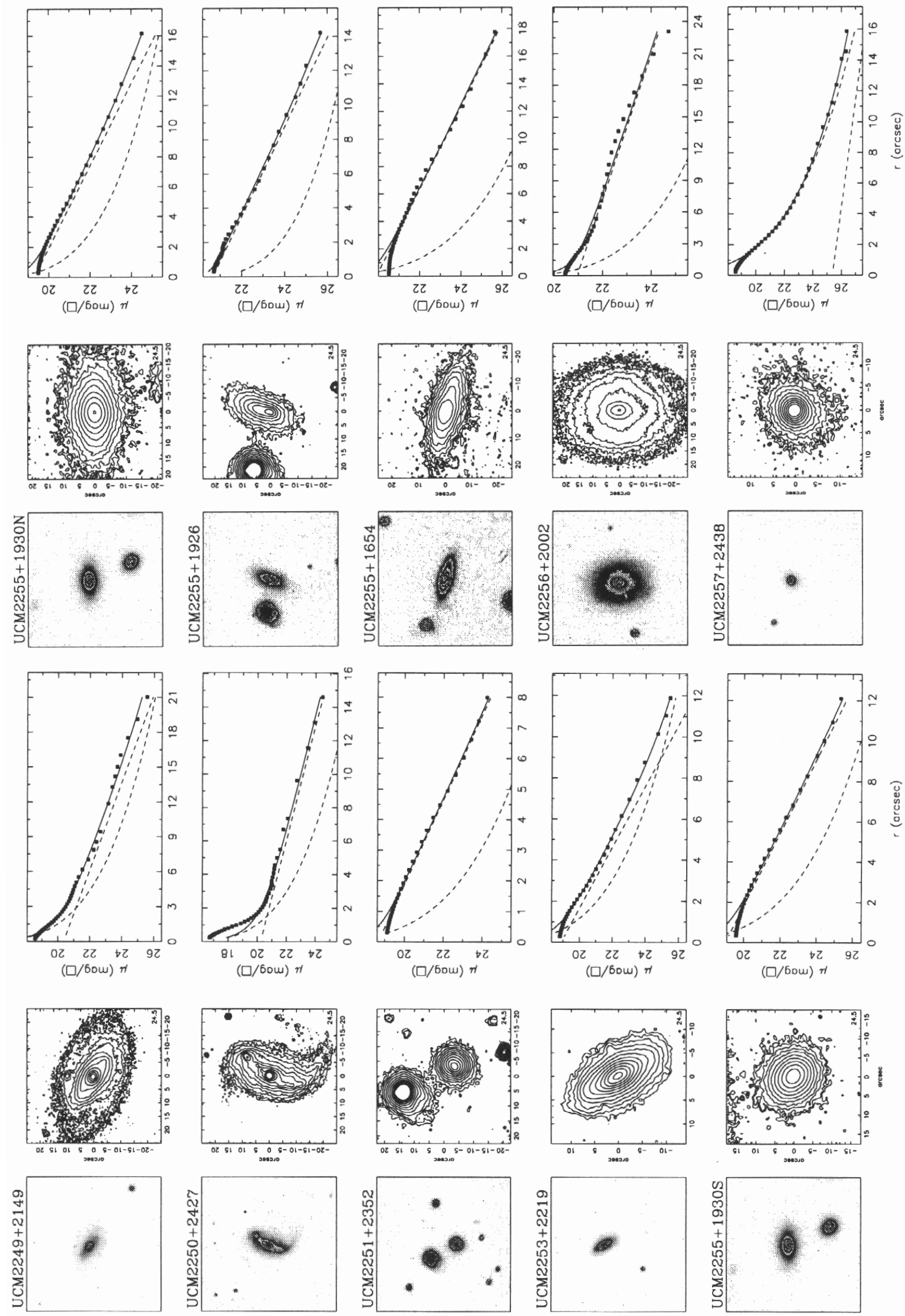

Fig. 1. continued 

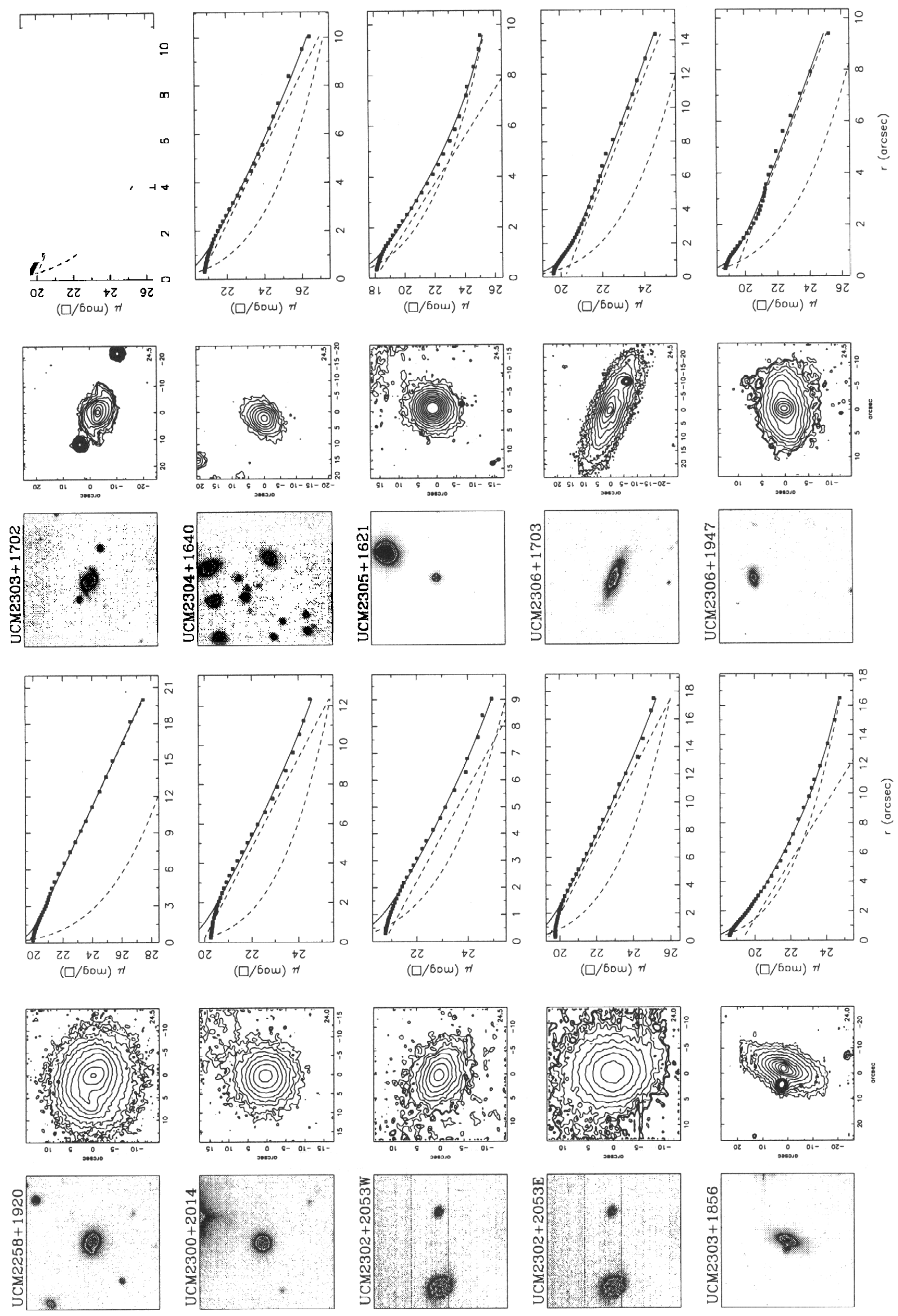

Fig. 1. continued 
A.G. Vitores et al.: Photometric and morphological analysis of UCM galaxies. I.
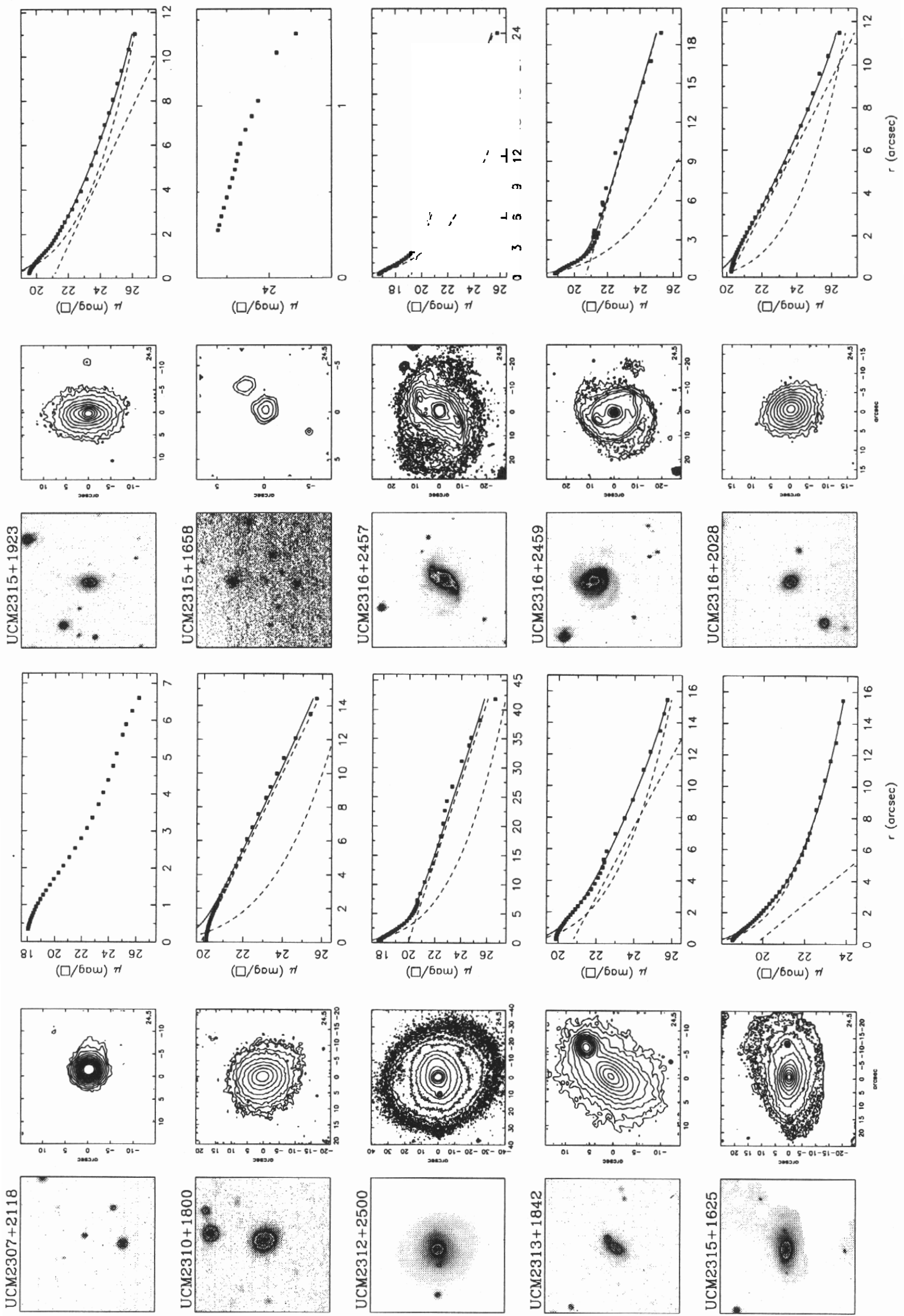

Fig. 1. continued 

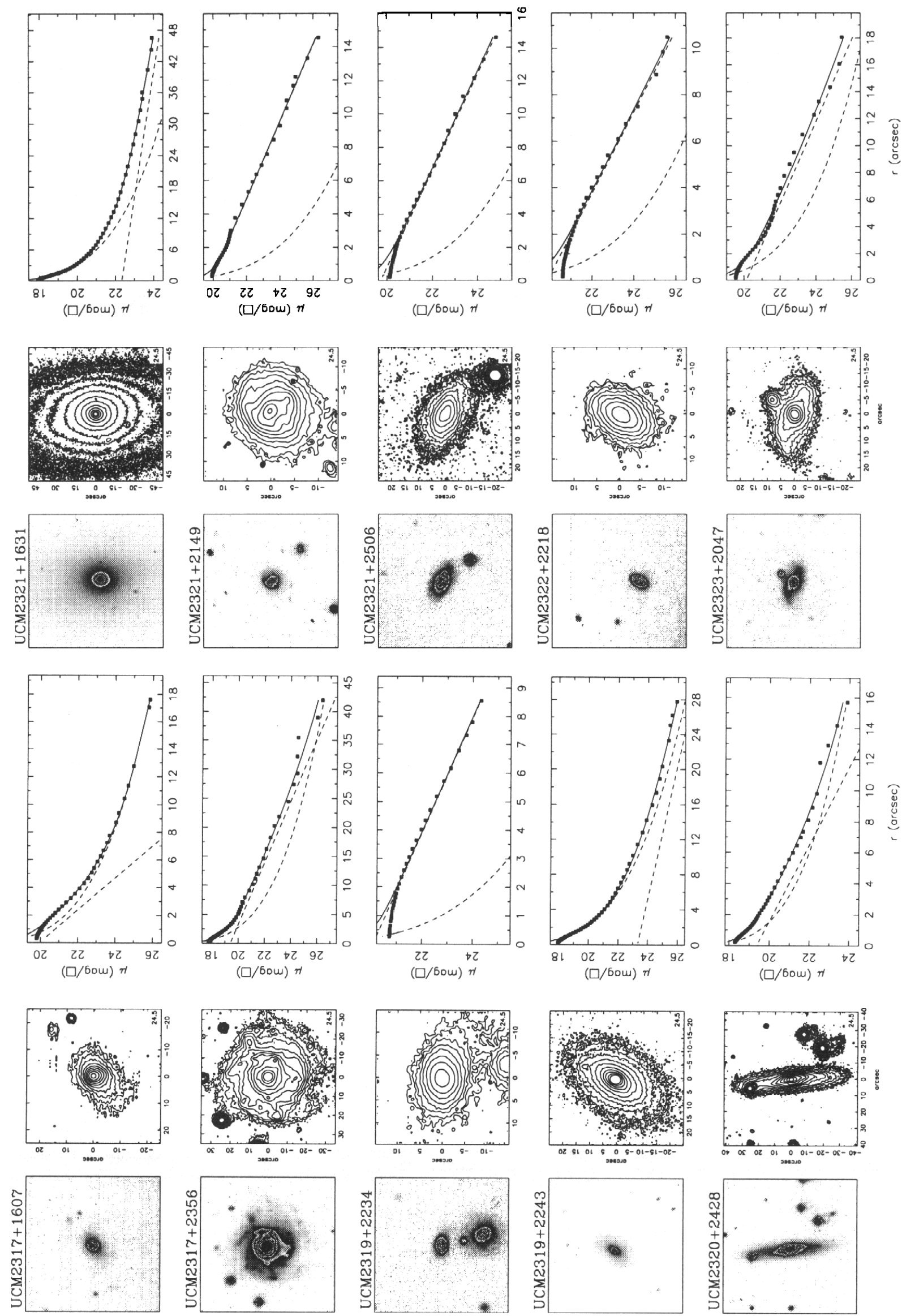

Fig. 1. continued 

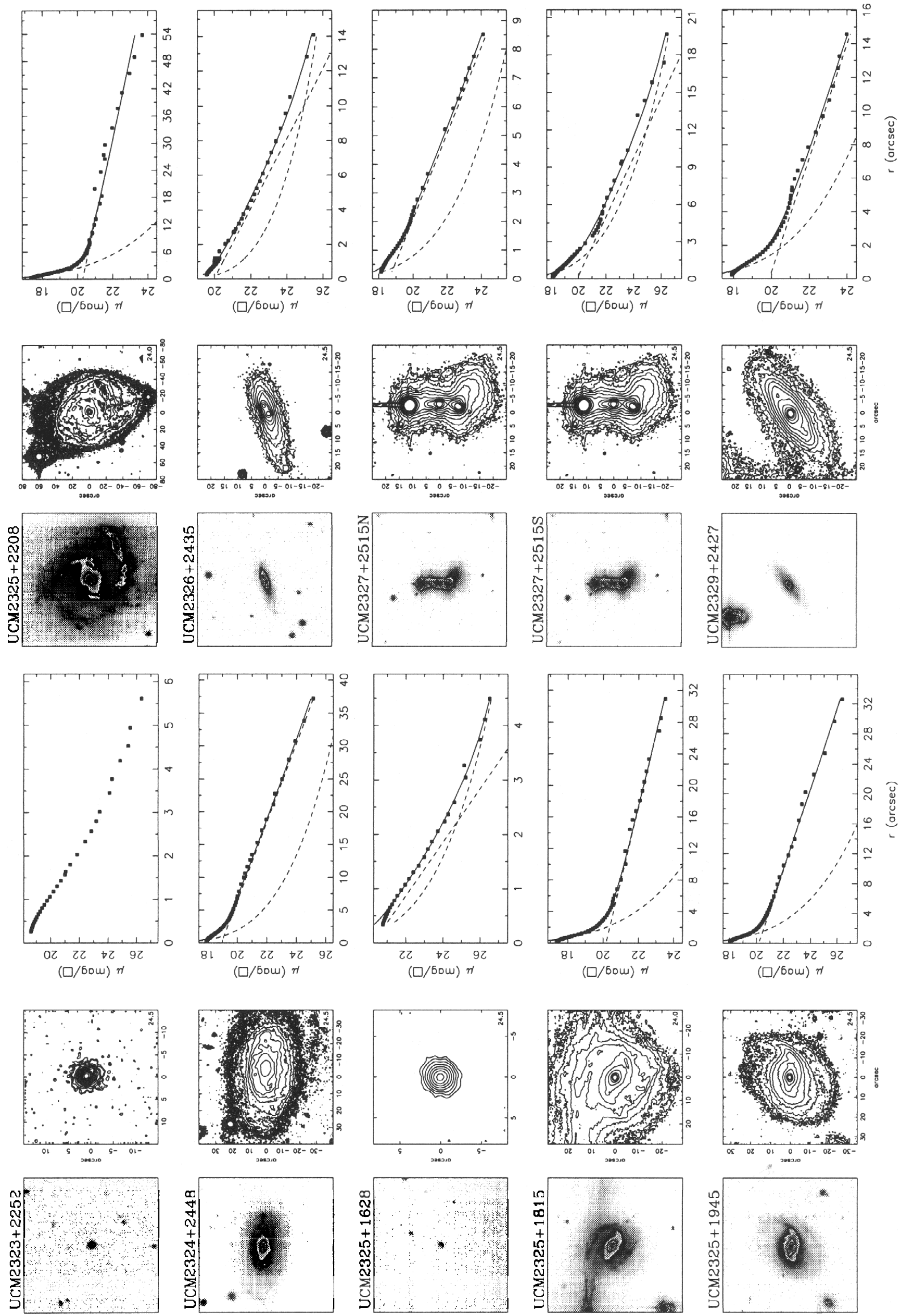

Fig. 1. continued 


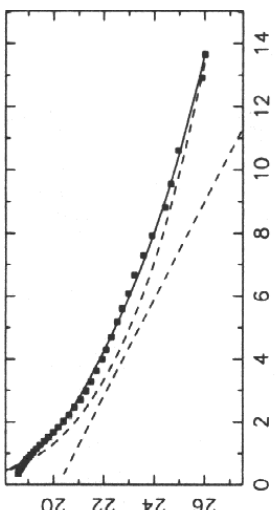

(口/50w) H
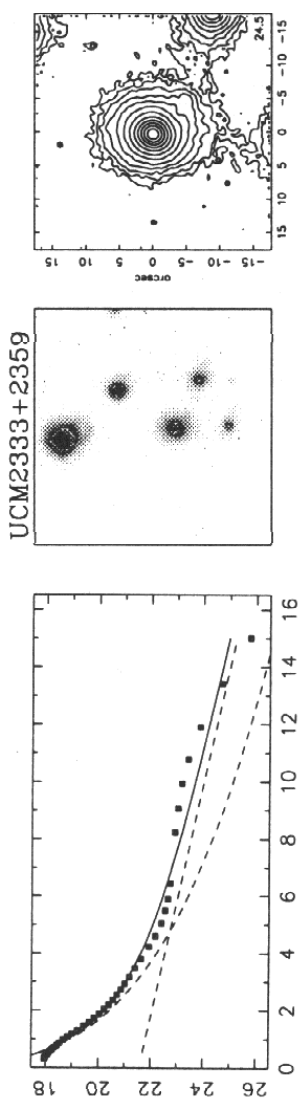

(ㅁ/6ow) $\pi$
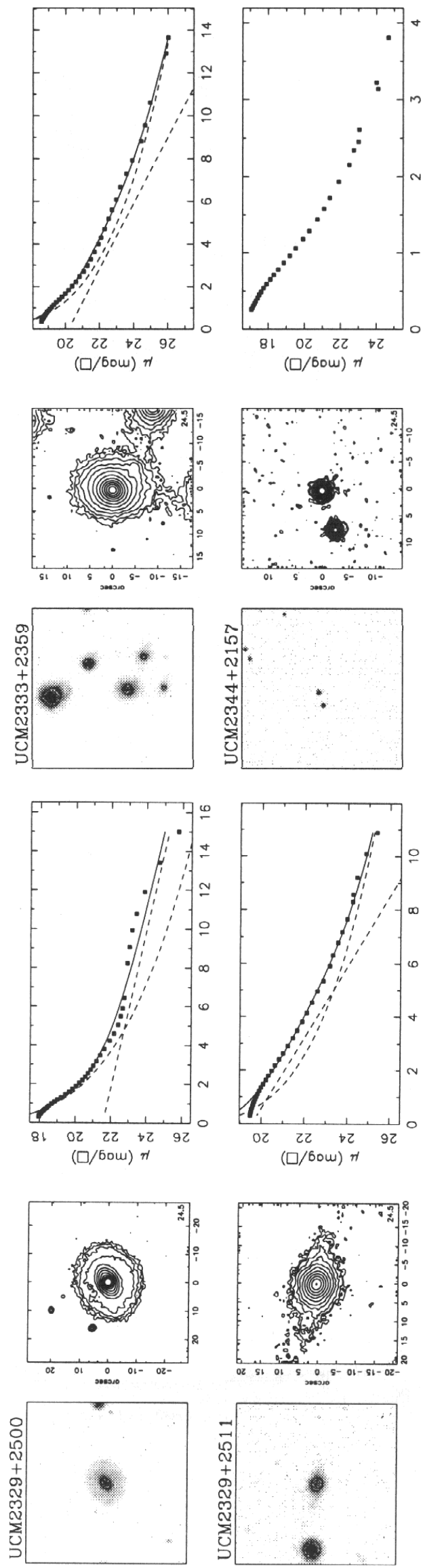
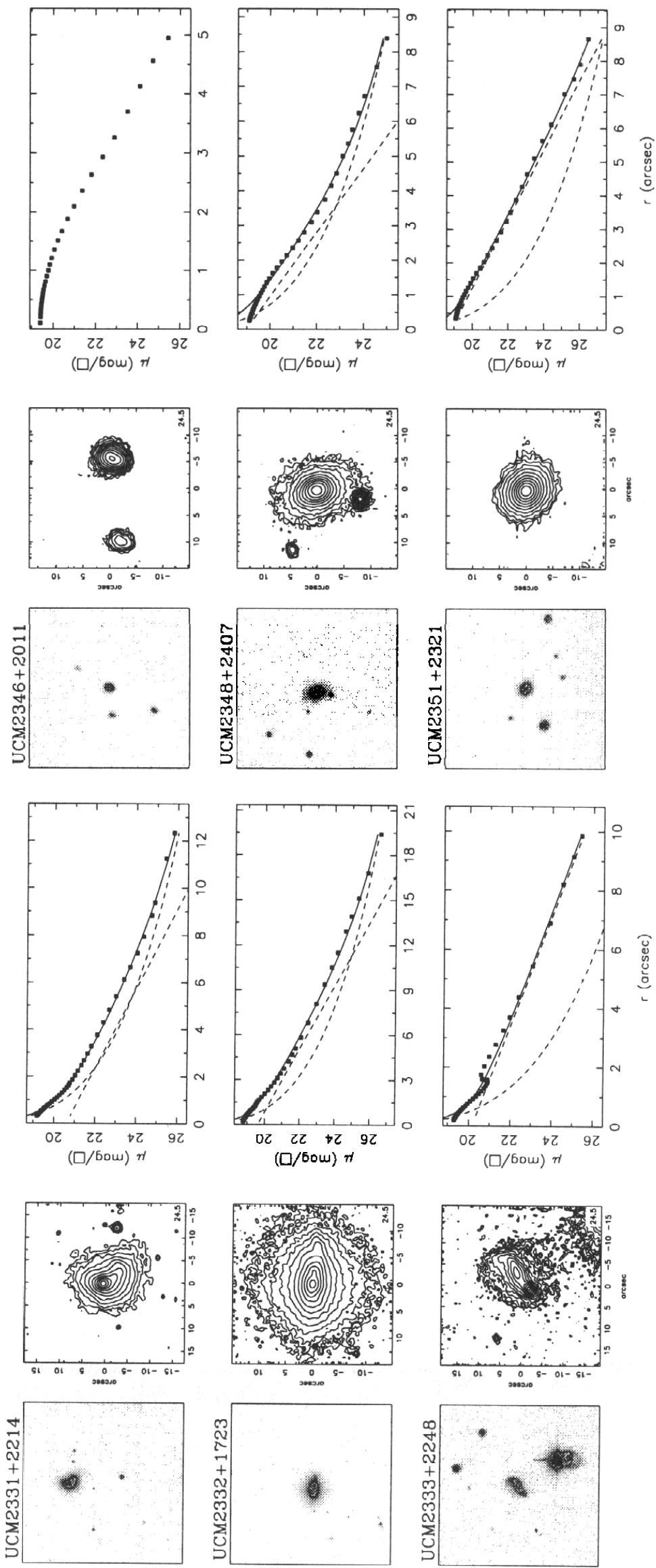

Fig. 1. continued 
A.G. Vitores et al.: Photometric and morphological analysis of UCM galaxies. I.
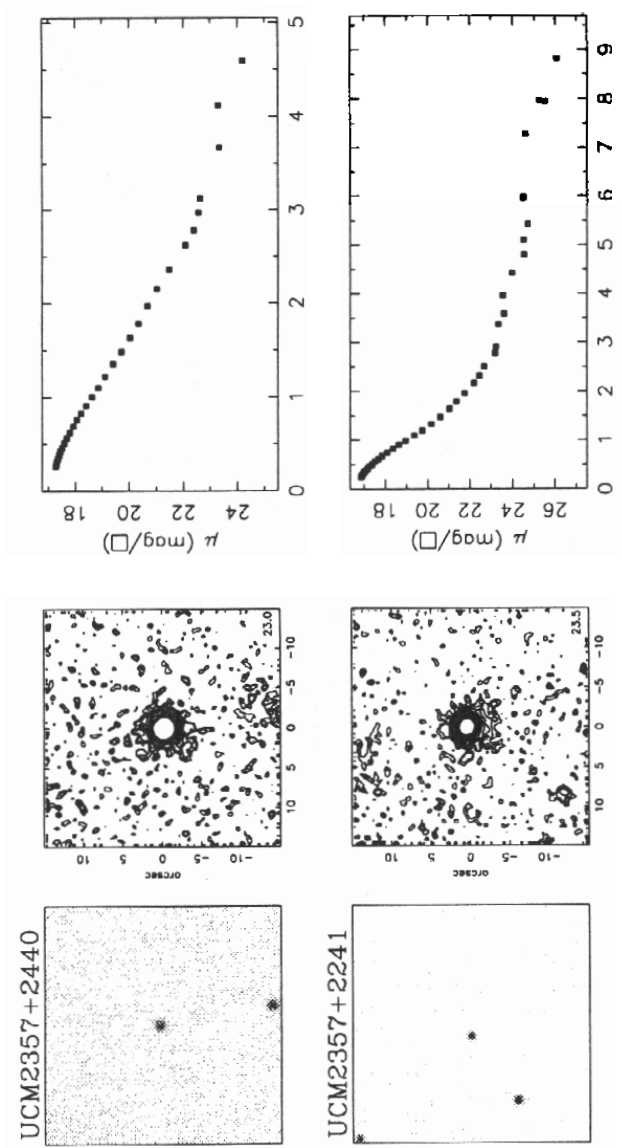

Fig. 1. continued 